\title{
Second order transport from anomalies
}

\author{
Sayantani Bhattacharyya, ${ }^{a}$ Justin R. David ${ }^{b}$ and Somyadip Thakur $^{b}$ \\ ${ }^{a}$ Physics Department, Ramkrishna Mission Vivekananda University, \\ Belur Math, Howrah 711202, India \\ ${ }^{b}$ Centre for High Energy Physics, Indian Institute of Science, \\ C.V. Raman Avenue, Bangalore 560012, India \\ E-mail: sayanta@rkmvu.ac.in, justin@cts.iisc.ernet.in, \\ somyadip@cts.iisc.ernet.in
}

ABSTRACT: We study parity odd transport at second order in derivative expansion for a non-conformal charged fluid. We see that there are 27 parity odd transport coefficients, of which 12 are non-vanishing in equilibrium. We use the equilibrium partition function method to express 7 of these in terms of the anomaly, shear viscosity, charge diffusivity and thermodynamic functions. The remaining 5 are constrained by 3 relations which also involve the anomaly. We derive Kubo formulae for 2 of the transport coefficients and show these agree with that derived from the equilibrium partition function.

KEYwords: Gauge-gravity correspondence, AdS-CFT Correspondence, Holography and condensed matter physics (AdS/CMT)

ARXIV EPRINT: 1305.0340 


\section{Contents}

1 Introduction $\quad 1$

1.1 Summary of the results 3

2 Anomalous transport from equilibrium partition function 6

$\begin{array}{lll}2.1 & \text { The equilibrium partition function method } & 7\end{array}$

2.2 Partition function analysis: parity-odd sector at second order 9

$\begin{array}{lll}3 & \text { Kubo formula for the transport coefficients } \Phi_{1}, \Phi_{2} & 17\end{array}$

$\begin{array}{lll}4 & \text { Chiral shear waves } & 21\end{array}$

5 Conclusions $\quad 24$

A Classification of parity odd data at 2nd order in derivatives $\quad 25$

$\begin{array}{ll}\text { B Consistency of the fluid profiles at the linear order } & 27\end{array}$

\section{Introduction}

Fluid dynamics is an effective description of near-equilibrium systems. Properties of a fluid system are always slowly varying compared to some intrinsic length scale, for example a mean free path. This length scale is determined by the details of the underlying microscopic theory. The fundamental variables of fluid dynamics are local velocities $u^{\mu}(x)$, temperature $T(x)$, and all the other conserved charges or their chemical potentials $\mu^{a}(x)$. The conservation equations for the stress tensor $T^{\mu \nu}$ and the other conserved currents $J^{a \mu}$ govern the time evolution of fluid dynamics.

The stress tensor and the conserved currents are related to the fluid variables $\left\{u^{\mu}, T, \mu^{a}\right\}$ via constitutive relations. Since fluid systems are always slowly varying it is appropriate to organize the constitutive relations in terms of a derivative expansion of the fluid variables. At every order in the derivative expansion, the independent terms of the constitutive relation are constructed out of the independent derivatives of the fluid variables. The independent terms in the constitutive relation are multiplied by coefficients which are functions of temperature and chemical potentials. These coefficients are called transport coefficients. In this paper we will study the transport coefficients that occur in the parity odd sector, at second order in the derivative expansion. These are terms constructed out of various derivatives in the fluid variables which are odd under parity. We will consider relativistic fluid systems with one additional conserved current.

It is usually difficult to compute transport coefficients from the microscopic theory and they are generically determined from experiments. However parity odd transport 
coefficients which occur at the first order in the derivative expansion have been related to quantum anomalies of the microscopic theory [1-8]. ${ }^{1}$ Our goal is to see if a similar phenomenon occurs at the second order in the derivative expansion of the constitutive relations. One motivation to consider second order fluid dynamics is that first order fluid dynamics is known to have problems with causality and numerical stability. Transport coefficients which occur at second order provide important constraints for spectral densities through sum rules [10]. Parity odd transport coefficients at second order affect the dispersion relation of chiral modes [11]. ${ }^{2}$ This phenomenon has important experimental consequences like the spatial separation of particles of different chirality. In the relativistic context this phenomenon was first observed holographically [12] and then understood due to the presence of a parity odd transport coefficient at second order by [13].

Parity odd transport coefficients at second order has been studied earlier in [13] for conformal fluids. They used the principle that parity odd terms which are even under timereversal invariance should not contribute to local entropy production. With this principle they could constrain these transport coefficients and determine some of them. We will use the method developed in $[14,15]$ to determine and constrain the parity odd transport coefficients. We consider non-conformal fluids in $3+1$ dimensions which admits one anomalous charge current. This method is based on the requirement that the fluid equations have to be consistent with the existence of an equilibrium partition function. Therefore the approach first relies on the physical requirement of the existence of equilibrium. More precisely:

- In a time independent background, that is a space-time metric with a time like Killing vector and background gauge fields independent of the time direction, any fluid equation will admit a time independent solution.

The second assumption is:

- The stress tensor and the charge current evaluated on this time independent solution can be obtained from the partition function by varying it with respect to the background metric and the gauge field.

This method is implemented as follows.

1. We first classify all the parity odd transport coefficients till the second order in the derivative expansion of the stress tensor and the charge current using symmetries.

2. We then evaluate the stress tensor and the charge current on the equilibrium fluid configuration to the second order in the derivative expansion.

3. The equilibrium partition function is written to the second order in derivative expansion taking all the parity odd terms into consideration. This is also done based on symmetries.

\footnotetext{
${ }^{1}$ See [9] for a recent review with a complete list of references.

${ }^{2}$ We thank Yashodhan Hatwalne for bringing this reference to our attention.
} 
4. This stress tensor and the charge current obtained from the equilibrium partition function is required to agree with that obtained from the stress tensor evaluated on the equilibrium fluid configuration.

From the description of the method it is clear that only transport coefficients which do not vanish in the equilibrium fluid configuration will be constrained or determined. We will see that in total there are 27 parity odd transport coefficients. Out of these 12 do not vanish in the equilibrium fluid configuration. Among the 12, we determine 7 which we label as $\Phi_{i}, i=1, \cdots 6$ and $\Delta_{2}$. and show that they are related to the anomaly. The rest of the 5 are constrained by 3 relations. These relations also involve the anomaly. The results are summarized in (1.9) and (1.10). We will then derive Kubo formulae for two of the transport coefficients $\Phi_{1}, \Phi_{2}$ and show that it agrees with the equilibrium partition function method. The remaining transport coefficients seem to be related to three point functions.

The organization of the paper is as follows. In the rest of the introduction we summarize our main results. In section 2 we implement the method of [14] to relate the transport coefficients which do not vanish in equilibrium to the anomalies. In section 3 we use the Kubo formalism to derive two of the transport coefficients. In section 4 we study the effects of the second order transport coefficients on linearized dispersion relations. We also verify the relation between the transport coefficient $\Phi_{1}$ and the anomaly coefficient obtained using the holographic evaluation of this transport coefficient for the case of $\mathcal{N}=$ 4 Yang-Mills. Using holography we also show that for the conformal case of $\mathcal{N}=4$ Yang-Mills the transport coefficient $\Phi_{12}$ vanishes. In appendix A we discuss some of the details involving the classification of the parity odd data at second order in derivatives. In appendix B we show the consistency of the velocity profile used to derive the Kubo formulae for transport coefficients.

\subsection{Summary of the results}

As we mentioned earlier, the aim of this note is to constrain the parity odd second order transport coefficients of an anomalous charged fluid in the presence of background electric and magnetic fields. To define the transport coefficients unambiguously we first must have an unambiguous definition of fluid variables, that is the velocities, the temperature and the chemical potentials. $\left\{u^{\mu}, T, \mu\right\}$. We will work in Landau frame which is defined by the following two conditions for the charged fluid.

$$
J^{\mu} u_{\mu}=-q, \quad T^{\mu \nu} u_{\mu}=-E u^{\nu} .
$$

Let us now consider the expansion of the charge current $J^{\mu}$ and the stress tensor $T^{\mu \nu}$ in terms of the number of space time derivatives. This is given by

$$
J^{\mu}=J_{(0)}^{\mu}+J_{(1)}^{\mu}+\cdots, \quad T^{\mu \nu}=T_{(0)}^{\mu \nu}+T_{(1)}^{\mu \nu}+\cdots,
$$

where the subscript $(i)$ refer to the number of space-time derivatives. The terms $J_{(i)}^{\mu}$ and $T_{(i)}^{\mu \nu}$ for $i \neq 0$ are all perpendicular to the velocity $u^{\mu}$. The equations of motion for the fluid 


\begin{tabular}{|c|c|c|c|}
\hline $\begin{array}{c}\text { Scalars } \\
(1)\end{array}$ & Vectors & Pseudo Vectors & Tensors \\
\hline$\Theta=\nabla_{\mu} u^{\mu}$ & $(3)$ & $(2)$ & $(1)$ \\
& $u^{\mu} \nabla_{\mu} u_{\nu}$ & $l^{\mu}=\epsilon^{\mu \nu \alpha \beta} u_{\nu} \partial_{\alpha} u_{\beta}$ & $\sigma_{\mu \nu}=\nabla_{\langle\mu} u_{\nu\rangle}$ \\
& $P^{\mu \nu} \nabla_{\nu} \nu$ & $B^{\mu}=\frac{1}{2} \epsilon^{\mu \nu \alpha \beta} u_{\nu} \mathcal{F}_{\alpha \beta}$ & \\
& $V^{\mu}=\left(\frac{E^{\mu}}{T}-P^{\mu \nu} \nabla_{\nu} \nu\right)$ & & \\
\hline
\end{tabular}

Table 1. Data at 1 st order in derivative.

in the presence of external electromagnetic field are given by the following conservation laws

$$
\begin{aligned}
\nabla_{\mu} T^{\mu \nu} & =\mathcal{F}^{\nu \mu} J_{\mu}+\frac{c_{m}}{2} \nabla_{\mu}\left[\epsilon^{\alpha \beta \gamma \delta} \mathcal{F}_{\alpha \beta} R_{\gamma \delta}^{\mu \nu}\right], \\
\nabla_{\mu} J^{\mu} & =-\frac{C}{8} \epsilon^{\alpha \beta \gamma \delta} \mathcal{F}_{\alpha \beta} \mathcal{F}_{\gamma \delta}+\frac{c_{m}}{4} \epsilon^{\alpha \beta \gamma \delta} R_{\nu \alpha \beta}^{\mu} R_{\mu \gamma \delta}^{\nu} \\
& =C E_{\mu} B^{\mu}+\frac{c_{m}}{4} \epsilon^{\alpha \beta \gamma \delta} R_{\nu \alpha \beta}^{\mu} R_{\mu \gamma \delta}^{\nu} .
\end{aligned}
$$

Here $E_{\mu}=\mathcal{F}_{\mu \nu} u^{\nu}, B^{\mu}=\frac{1}{2} \epsilon^{\mu \nu \alpha \beta} u_{\nu} \mathcal{F}_{\alpha \beta}$ and $C$ is the gauge anomaly coefficient and $c_{m}$ is the coefficient of the mixed gauge-gravitational anomaly. Note that the terms proportional to the mixed anomaly are fourth order in derivatives, therefore they do not affect the analysis of the equations of motion to 2 nd order in derivatives. However the gravitational anomaly does enter the discussion of the equilibrium partition function at the first order in the derivative expansion [16].

We will now state the known results for the form of the stress tensor and the current up to first order in the derivative expansion. For $i=0$, the part with no space-time derivative the stress tensor and current is completely determined by thermodynamics. At first order in derivative expansion, that is $i=1$, the form of the current and stress tensor is explicitly known [1, 14]. This form is consistent with all physical requirements in the presence of anomaly as well as an external electromagnetic field $[1,14]$. The final result for the stress tensor and the charge current to first order in derivatives is given by

$$
\begin{aligned}
{\left[T_{(0)}\right]_{\mu \nu} } & =(E+P) u_{\mu} u_{\nu}+P G_{\mu \nu} \\
{\left[T_{(1)}\right]_{\mu \nu} } & =-2 \eta \sigma^{\mu \nu}-\zeta \Theta P^{\mu \nu} \\
J_{(0)}^{\mu} & =q u^{\mu} \\
J_{(1)}^{\mu} & =\Delta V^{\mu}+\xi_{l} l^{\mu}+\xi_{B} B^{\mu} .
\end{aligned}
$$

Here $E$ is the energy density, $P$ the pressure and $q$, the charge density and $G_{\mu \nu}$ is the background metric. The variables $\Theta, \sigma^{\mu \nu}, V^{\mu}, l^{\mu}$ and $B^{\mu}$ are all on-shell independent terms which are first order in derivatives. These are defined in table 1 . The variables $\eta, \zeta, \Delta, \xi_{l}$ and $\xi_{B}$ refer to the first order transport coefficients. Throughout this paper, the symbol $A_{\langle\mu \nu\rangle}$ on any tensor $A_{\mu \nu}$ denotes the projected, traceless, symmetric part of the tensor.

$$
A_{\langle\mu \nu\rangle}=P_{\mu}^{\alpha} P_{\nu}^{\beta}\left(\frac{A_{\alpha \beta}+A_{\beta \alpha}}{2}-\frac{P^{\gamma \theta} A_{\gamma \theta}}{3} G_{\alpha \beta}\right)
$$




\begin{tabular}{|c|c|c|}
\hline $\begin{array}{c}\text { Pseudo-scalars } \\
(6)\end{array}$ & Pseudo Vectors & Pseudo-tensors \\
\hline $\mathcal{S}_{1}=l^{\mu} \partial_{\mu} \nu$ & $(9)$ & $(12)$ \\
$\mathcal{S}_{2}=B^{\mu} \partial_{\mu} \nu$ & $\mathcal{V}_{(1)}^{\mu}=\epsilon^{\mu \nu \alpha \beta} u_{\nu} B_{\alpha} l_{\beta}$ & $\tau_{\mu \nu}^{(1)}=\nabla_{\langle\mu} l_{\nu\rangle}$ \\
$\mathcal{S}_{3}=l^{\mu} \partial_{\mu} T$ & $\epsilon^{\mu \nu \alpha \beta} u_{\nu}\left(\partial_{\alpha} \nu\right)\left(\partial_{\alpha} T\right)$ & $\tau_{\mu \nu}^{(2)}=\nabla_{\langle\mu} B_{\nu\rangle}$ \\
$\mathcal{S}_{4}=B^{\mu} \partial_{\mu} T$ & $\mathcal{V}_{(3)}^{\mu}=\Theta l^{\mu}$ & $\tau_{\mu \nu}^{(3)}=l_{\langle\mu} \partial_{\nu\rangle} \nu$ \\
$\mathcal{S}_{5}=l^{\mu} V_{\mu}$ & $\mathcal{V}_{(4)}^{\mu}=\epsilon^{\mu \nu \lambda \sigma} u_{\nu} \nabla_{\lambda} V_{\sigma}$ & $\tau_{\mu \nu}^{(4)}=B_{\langle\mu} \partial_{\nu\rangle} \nu$ \\
$\mathcal{S}_{6}=B^{\mu} V_{\mu}$ & $\mathcal{V}_{(5)}^{\mu}=\sigma_{\nu}^{\mu} l^{\nu}$ & $\tau_{\mu \nu}^{(5)}=l_{\langle\mu} \partial_{\nu\rangle} T$ \\
& $\mathcal{V}_{(6)}^{\mu}=\Theta B^{\mu}$ & $\tau_{\mu \nu}^{(6)}=B_{\langle\mu} \partial_{\nu\rangle} T$ \\
& $\mathcal{V}_{(7)}^{\mu}=\sigma_{\nu}^{\mu} B^{\nu}$ & $\tau_{\mu \nu}^{(7)}=l_{\langle\mu} V_{\nu\rangle}$ \\
& $\mathcal{V}_{(8)}^{\mu}=\epsilon^{\mu \nu \alpha \beta} u_{\nu}\left(\partial_{\alpha} T\right) V_{\beta}$ & $\tau_{\mu \nu}^{(8)}=B_{\langle\mu} V_{\nu\rangle}$ \\
& $\mathcal{V}_{(9)}^{\mu}=\epsilon^{\mu \nu \alpha \beta} u_{\nu}\left(\partial_{\alpha} \nu\right) V_{\beta}$ & $\tau_{\mu \nu}^{(9)}=u_{\theta} \sigma_{\langle\mu \alpha}\left(\partial_{\beta} T\right) \epsilon^{\theta \alpha \beta}{ }_{\nu\rangle}$ \\
& \multicolumn{2}{|c}{$\tau_{\mu \nu}^{(10)}=u_{\theta} \sigma_{\langle\mu \alpha}\left(\partial_{\beta} \nu\right) \epsilon^{\theta \alpha \beta}{ }_{\nu\rangle}$} \\
& \multicolumn{2}{|c|}{$\tau_{\mu \nu}^{(11)}=u_{\theta} \sigma_{\langle\mu \alpha} V_{\beta} \epsilon_{\nu\rangle}^{\theta \alpha}$} \\
& $\tau_{\mu \nu}^{(12)}=u_{\theta} \nabla_{\beta} \sigma_{\langle\mu \alpha} \epsilon^{\theta \alpha \beta}{ }_{\nu\rangle}$ \\
\hline
\end{tabular}

Table 2. Parity odd data at 2nd order in derivatives.

and $P^{\mu \nu}$ is the projector

$$
P^{\mu \nu}=u^{\mu} u^{\nu}+G^{\mu \nu} .
$$

The variable $\nu$ is related to the chemical potential by

$$
\nu=\frac{\mu}{T} \text {. }
$$

Let us now proceed to the stress tensor and the charge current at second order in derivatives which we denote as $\left[T_{(2)}\right]_{\mu \nu}$ and $J_{(2)}^{\mu}$. Purely from symmetry considerations, the number of independent transport coefficients upto second order is equal to total number of possible scalars which appear in the trace of the stress tensor, together with the number of possible vectors which appear in the current and the possible symmetric traceless tensors which appear in the traceless part of the stress tensor. But not all of them are independent, they can be related using the equations of motion given in (1.3). In table 2 we have listed all the parity odd and on-shell independent scalars, vectors and tensors containing two space-time derivatives. Some of the details that went into this classification is discussed in appendix A.

From the table it can be seen that at second order in derivatives there are 27 parity odd transport coefficients which appear in the current and the stress tensor. Therefore the most general parity odd contributions at second order in the stress tensor and current can be parametrized as follows.

$$
\begin{aligned}
{\left[T_{(2)}\right]_{\mu \nu} } & =\sum_{i=1}^{12} \Phi_{i} \tau_{\mu \nu}^{(i)}+P_{\mu \nu}\left[\sum_{i=1}^{6} \chi_{i} \mathcal{S}_{i}\right] \\
J_{(2)}^{\mu} & =\sum_{i=1}^{9} \Delta_{i} \mathcal{V}_{(i)}^{\mu} .
\end{aligned}
$$


Where $\tau_{\mu \nu}^{(i)}, \mathcal{S}_{i}$ and $\mathcal{V}_{(i)}^{\mu}$ are defined in table 2. Our goal is to constrain the transport coefficients $\Phi_{i}, \chi_{i}$ and $\Delta_{i}$, using the existence of an equilibrium partition function. It can be shown among these 27 terms only 12 can be non-zero in a time independent equilibrium fluid configuration. These are the first 4 in the list of scalars $\mathcal{S}_{1} \cdots \mathcal{S}_{4}$, the first 2 in the list of vectors $\mathcal{V}_{(1)}^{\mu}, \mathcal{V}_{(2)}^{\mu}$ and the first 6 in the list of tensors $\tau_{\mu \nu}^{(1)} \cdots \tau_{\mu \nu}^{(6)}$. Therefore the analysis using the equilibrium partition function can be used to constrain the 12 transport coefficients multiplying these non-vanishing terms. These are $\chi_{1} \cdots \chi_{4}$ and $\Delta_{1}, \Delta_{2}$ and $\Phi_{1} \cdots \Phi_{6}$. The final result of this analysis is the following.

$$
\begin{gathered}
\Phi_{1}=\eta b_{1}, \quad \Phi_{2}=2 \eta b_{2}, \quad \Phi_{3}=\eta\left(\frac{\partial b_{1}}{\partial \nu}\right), \quad \Phi_{4}=2 \eta\left(\frac{\partial b_{2}}{\partial \nu}\right), \\
\Phi_{5}=\eta\left[-\frac{b_{1}}{T}+\frac{\partial b_{1}}{\partial T}\right], \quad \Phi_{6}=2 \eta\left[-\frac{b_{2}}{T}+\frac{\partial b_{2}}{\partial T}\right], \\
\Delta_{2}=-\frac{\Delta b_{1}}{2}, \\
T^{2} R_{1}\left[\chi_{3}-\frac{\zeta}{2}\left(\frac{\partial b_{1}}{\partial T}-\frac{2 b_{1}}{T}\right)\right]-R_{2}\left[\chi_{1}-\frac{\zeta}{2}\left(\frac{\partial b_{1}}{\partial \nu}-2 b_{2} T\right)\right]=0 \\
T^{2} R_{1}\left[\chi_{4}-\zeta\left(\frac{\partial b_{2}}{\partial T}-\frac{b_{2}}{T}\right)\right]+R_{2}\left[\chi_{2}-\zeta\left(\frac{\partial b_{2}}{\partial \nu}\right)\right]=0, \\
R_{1} T \Delta_{1}+\left[\chi_{2}-\zeta\left(\frac{\partial b_{2}}{\partial \nu}\right)\right]-\frac{q}{(E+P)}\left[\chi_{1}-\frac{\zeta}{2}\left(\frac{\partial b_{1}}{\partial \nu}-2 b_{2} T\right)\right]=0,
\end{gathered}
$$

where

$$
\begin{aligned}
b_{1} & =\frac{T^{3}}{E+P}\left(\frac{2 C \nu^{3}}{3}-4 C_{2} \nu\right), & b_{2} & =\frac{T^{2}}{E+P}\left(\frac{C \nu^{2}}{2}-C_{2}\right), \\
R_{1} & =\left(\frac{\partial P}{\partial E}\right)_{q}, & R_{2} & =\left(\frac{\partial P}{\partial q}\right)_{E} .
\end{aligned}
$$

Note that $C$ is the gauge anomaly coefficient and $C_{2}$ is related to the coefficient of the mixed gauge-gravitational anomaly $[16]$ by $^{3}$

$$
C_{2}=8 \pi^{2} c_{m}
$$

Therefore we see that the coefficients $\Phi_{1}, \cdots \Phi_{6}$ and $\Delta_{2}$ are determined in terms of the anomaly, the shear viscosity $\eta$, the charge diffusivity $\Delta$ and the thermodynamic functions $E, P, T, \nu$. The rest $\Delta_{1}, \chi_{1}, \cdots \chi_{4}$ are constrained by 3 relations which involve the anomaly and the bulk viscosity $\zeta$.

\section{Anomalous transport from equilibrium partition function}

In subsection 2.1 we will briefly outline the general procedure we use to relate parity odd transport at the second order in derivatives to the anomaly. This method has been

\footnotetext{
${ }^{3}$ The relation (1.11) is derived in [16] using properties of the partition function on cones. It is an equation that relates coefficients at different orders in derivative expansion. In our analysis we shall simply assume their result. We will subsequently see that this identification is also consistent with the analysis of [9] which studies the effect of gravitational anomalies on hydrodynamics.
} 
described and analyzed in detail by [14] and [15]. It relies on the analysis of the equilibrium partition function. Thus we will be able to constrain only those transport coefficients which do not vanish in the equilibrium configuration. This section also will serve to introduce the notation and conventions used in the paper. In subsection 2.2 we implement this method and derive the relations given in (1.9) and (1.10).

\subsection{The equilibrium partition function method}

We are interested in a fluid flow on a static background metric and a static external electromagnetic field. The most general static metric and gauge field can be written in the following form.

$$
d s^{2}=G_{\mu \nu} d x^{\mu} d x^{\nu}=-e^{2 \sigma}\left(d t+a_{i} d x^{i}\right)^{2}+g_{i j} d x^{i} d x^{j},
$$

Gauge Field : $\mathcal{A}_{\mu} d x^{\mu}$,

$\sigma, a_{i}, g_{i j}, \mathcal{A}_{0}$ and $\mathcal{A}_{\mu}$ are all slowly varying functions of the spatial co-ordinates $(\vec{x})$. Our notations are as follows:

- Greek indices run from 1 to 4.

- Latin indices run from 1 to 3 .

- All Greek indices are lowered or raised by the 4 dimensional metric $G_{\mu \nu}$ unless explicitly mentioned.

- All Latin indices are lowered or raised by the 3 dimensional metric $g_{i j}$ unless explicitly mentioned.

- $\bar{\nabla}_{\mu}$ is covariant derivative with respect to the metric $G_{\mu \nu}$ and $\nabla_{i}$ is the covariant derivative with respect to the metric $g_{i j}$.

- For any tensor $A_{\mu \nu}$ the notation $A_{\langle\mu \nu\rangle}$ denotes the traceless symmetric part of the tensor, projected in direction perpendicular to the fluid velocity.

$$
A_{\langle\mu \nu\rangle}=P_{\mu}^{\alpha} P_{\nu}^{\beta}\left(\frac{A_{\alpha \beta}+A_{\beta \alpha}}{2}-\frac{P^{\gamma \theta} A_{\gamma \theta}}{3} G_{\alpha \beta}\right)
$$

where $P_{\mu \nu} \equiv u_{\mu} u_{\nu}+G_{\mu \nu}$ is referred to as the projector.

Our basic assumption is that in such a background any fluid equation will admit a time independent solution. The stress tensor and the current, evaluated on this time independent solution, can be generated by varying the partition function of the system with respect to the background metric and gauge field. If $Z$ is the partition function at temperature $T_{0}$ then the stress tensor and the current evaluated on the equilibrium are 
given by the following formulae.

$$
\begin{aligned}
\left.T_{00}\right|_{\text {equilibrium }} & =-\frac{T_{0} e^{2 \sigma}}{\sqrt{-G}}\left[\frac{\delta Z}{\delta \sigma}\right]=\frac{T_{0}^{2}}{\sqrt{g}}\left[\frac{\delta Z}{\delta \bar{T}}\right], \\
\left.T_{0}^{i}\right|_{\text {equilibrium }} & =\frac{T_{0}}{\sqrt{-G}}\left[\frac{\delta Z}{\delta a_{i}}-A_{0} \frac{\delta Z}{\delta A_{i}}\right]=\frac{\bar{T}}{\sqrt{g}}\left[\frac{\delta Z}{\delta a_{i}}-A_{0} \frac{\delta Z}{\delta A_{i}}\right], \\
\left.T^{i j}\right|_{\text {equilibrium }} & =-\frac{2 T_{0}}{\sqrt{-G}} g^{i l} g^{j m}\left[\frac{\delta Z}{\delta g^{l m}}\right]=-\frac{2 \bar{T}}{\sqrt{g}} g^{i l} g^{j m}\left[\frac{\delta Z}{\delta g^{l m}}\right], \\
\left.J_{0}\right|_{\text {equilibrium }} & =-\frac{T_{0} e^{2 \sigma}}{\sqrt{-G}}\left[\frac{\delta Z}{\delta A_{0}}\right]=-\frac{e^{\sigma}}{\sqrt{g}}\left[\frac{\delta Z}{\delta \bar{\nu}}\right], \\
\left.J^{i}\right|_{\text {equilibrium }} & =\frac{T_{0}}{\sqrt{-G}}\left[\frac{\delta Z}{\delta A_{i}}\right]=\frac{\bar{T}}{\sqrt{g}}\left[\frac{\delta Z}{\delta A_{i}}\right]
\end{aligned}
$$

where

$$
\bar{T}=T_{0} e^{-\sigma}, \quad \bar{\nu}=\frac{A_{0}}{T_{0}}, \quad A_{i}=\mathcal{A}_{i}-A_{0} a_{i} .
$$

The strategy we will adopt to constrain the parity odd coefficients which occur at the second order is the following:

1. Write down the most general partition function upto a given order in derivative expansion and consistent with all the symmetries. It will be a function of $a_{i}, \sigma$ and $\mathcal{A}_{i}$ and their derivatives.

2. Vary the partition function $Z$, to obtain the most general possible expression for $\left.T^{\mu \nu}\right|_{\text {equilibrium }}$ and $\left.J^{\mu}\right|_{\text {equilibrium}}$.

3. Parametrize the most general possible fluid stress tensor and current up to some given order in derivative expansion using symmetries. This will give the maximum number of independent transport coefficients possible constrained only by symmetry. We have stated the results of this analysis already in equations (1.4) and (1.8).

4. Evaluate the most general fluid stress tensor and current on the equilibrium solution. The final outcome will contain some of the unknown transport coefficients.

5. Equate the final outcome of the previous step with the stress tensor and current we have already obtained by varying the partition function.

6. This will express the transport coefficients which appear in the stress tensor and the current evaluated in equilibrium, in terms of the coefficients appearing in the partition function.

7. Eliminating the coefficients which appear in the partition function results in the desired relations among the transport coefficients.

One might wonder that to execute the fourth step, the equilibrium solution for the velocity, temperature and the other fluid variables needs to be independently found. But as it has been explained in [14] using this method one can perturbatively determine both the solution and the transport coefficients in terms of the free functions appearing in the partition function. 


\subsection{Partition function analysis: parity-odd sector at second order}

In this subsection we shall apply the general procedure described in 2.1 to the particular case of the parity-odd sector of the charged fluid at second order in derivative expansion. The first contribution to the parity-odd sector comes at first order in derivative expansion. This has been analyzed in detail in section 3 of [14]. We will not repeat the first order computation here but we will extensively use their result.

Stress tensor and current from the partition function. Since the transport coefficients we are interested in belong to the parity-odd sector, we will restrict our attention to only the parity odd part of the partition function. The partition function $Z_{(2)}$ at second order in derivatives is a gauge invariant scalar functional of the background metric and gauge-fields. ${ }^{4}$ Hence we need to list all possible parity odd scalars that can be constructed from the metric functions and gauge fields that contain two space-derivatives. Note that since all the functions are time independent no time derivatives occur. There are four such scalars:

1. $\epsilon^{i j k} \partial_{i} \bar{\nu} f_{j k}$,

2. $\epsilon^{i j k} \partial_{i} \bar{T} f_{j k}$,

3. $\epsilon^{i j k} \partial_{i} \bar{\nu} F_{j k}$,

4. $\epsilon^{i j k} \partial_{i} \bar{T} F_{j k}$,

where $\bar{\nu}=\frac{A_{0}}{T_{0}}, \quad \bar{T}=T_{0} e^{-\sigma}, \quad A_{i}=\mathcal{A}_{i}-A_{0} a_{i}$ and $f_{j k}=\partial_{j} a_{k}-\partial_{k} a_{j}, \quad F_{j k}=\partial_{j} A_{k}-\partial_{k} A_{j}$.

Therefore naively, the parity odd second order partition function at two derivative order can have 4 free parameters, but two of them can be related by total derivatives. Ignoring the total derivative terms the most general second order partition function can be written as

$$
Z_{(2)}=\int \sqrt{g}\left[M_{1}(\bar{T}, \bar{\nu}) \epsilon^{i j k} \partial_{i} \bar{\nu} F_{j k}+T_{0} M_{2}(\bar{T}, \bar{\nu}) \epsilon^{i j k} \partial_{i} \bar{\nu} f_{j k}\right] .
$$

Varying this partition function with respect to the metric and the gauge fields and using (2.2) we get the following second order correction to the stress tensor and charge current.

$$
\begin{aligned}
{\left[\Pi^{(2)}\right]_{00} } & =T_{0}^{2}\left[\left(\frac{\partial M_{1}}{\partial \bar{T}}\right) \epsilon^{i j k} \partial_{i} \bar{\nu} F_{j k}+T_{0}\left(\frac{\partial M_{2}}{\partial \bar{T}}\right) \epsilon^{i j k} \partial_{i} \bar{\nu} f_{j k}\right], \\
{\left[\Pi^{(2)}\right]_{0}^{i} } & =2 T_{0} \bar{T}\left(\frac{\partial M_{2}}{\partial \bar{T}}-\bar{\nu} \frac{\partial M_{1}}{\partial \bar{T}}\right) \epsilon^{i j k}\left(\partial_{j} \bar{T}\right)\left(\partial_{k} \bar{\nu}\right), \\
{\left[\Pi^{(2)}\right]^{i j} } & =0, \\
{\left[j^{(2)}\right]_{0} } & =\frac{T_{0}}{\bar{T}}\left[\left(\frac{\partial M_{1}}{\partial \bar{T}}\right) \epsilon^{i j k} \partial_{i} \bar{T} F_{j k}+T_{0}\left(\frac{\partial M_{2}}{\partial \bar{T}}\right) \epsilon^{i j k} \partial_{i} \bar{T} f_{j k}\right], \\
{\left[j^{(2)}\right]^{i} } & =2 \bar{T}\left(\frac{\partial M_{1}}{\partial \bar{T}}\right) \epsilon^{i j k}\left(\partial_{j} \bar{T}\right)\left(\partial_{k} \bar{\nu}\right) .
\end{aligned}
$$

\footnotetext{
${ }^{4}$ Though the system is anomalous, all the effects of anomaly i.e. the anomalous transformation property of the partition function under the gauge transformation is accounted by the first order part $Z_{(1)}$. Therefore in $Z_{(2)}$ we need to consider only gauge invariant scalars.
} 
It is important to note the third equation in (2.3). The fact that the spatial components of the equilibrium stress tensor vanish at second order will serve as an important constraint in determining the transport coefficients.

Stress tensor from fluid dynamics. We have to evaluate the fluid dynamical stress tensor on the equilibrium, that is a time independent solution in the given static background metric and gauge field. This equilibrium solution for the velocity field, temperature or chemical potential in terms of the background metric and gauge field can also be expanded in terms of derivatives. We shall use the following notation.

$$
\begin{aligned}
\left.u^{\mu}\right|_{e q} & =\bar{u}^{\mu}+\delta u_{(1)}^{\mu}+\delta u_{(2)}^{\mu}+\cdots, \\
\left.T\right|_{e q} & =\bar{T}+\delta T_{(1)}+\delta T_{(2)}+\cdots, \\
\left.\nu\right|_{e q} & =\bar{\nu}+\delta \nu_{(1)}+\delta \nu_{(2)}+\cdots,
\end{aligned}
$$

where $\delta u_{(i)}^{\mu}, \delta T_{(i)}$ and $\delta \nu_{(i)}$ are $i$ th corrections to the zeroth order equilibrium solution containing $i$ derivatives on the background data. Now we have to substitute (2.4) in fluid stress tensor and current given in (1.4)) and extract the part that will be parity odd and which involve exactly two derivatives on the background data. This is the part which have to be equated with (2.3).

From the analysis done in [14] we know that ${ }^{5}$

$$
\begin{aligned}
\bar{u}^{\mu} & =e^{-\sigma}\{1,0,0,0\}, \quad \bar{T}=T_{0} e^{-\sigma}, \quad \bar{\nu}=\frac{A_{0}}{T_{0}}, \\
{\left[\delta u_{(1)}\right]_{0} } & =0, \quad \delta T_{(1)}=0, \quad \delta \nu_{(1)}=0, \\
{\left[\delta u_{(1)}\right]^{0} } & =-a_{i}\left[\delta u_{(1)}\right]^{i}, \\
{\left[\delta u_{(1)}\right]^{i} } & =\left(\frac{b_{1}}{2}\right) \bar{l}^{i}+b_{2} \bar{B}^{i}, \\
{\left[\delta u_{(1)}\right]_{i} } & =g_{i j}\left[\delta u_{(1)}\right]^{j},
\end{aligned}
$$

where

$$
\begin{aligned}
F_{j k} & \equiv \partial_{j} A_{k}-\partial_{k} A_{j}, \\
\bar{l}^{i} & =-\frac{e^{\sigma}}{2} \epsilon^{i j k} f_{j k}, \\
\bar{B}^{i} & =\frac{1}{2}\left(\epsilon^{i j k} F_{j k}+A_{0} \epsilon^{i j k} f_{j k}\right)=\frac{1}{2} \epsilon^{i j k} F_{j k}-\bar{T} \bar{\nu} \bar{l}^{i}, \\
b_{1} & =\frac{T^{3}}{E+P}\left(\frac{2 C \nu^{3}}{3}-4 C_{2} \nu\right), \\
b_{2} & =\frac{T^{2}}{E+P}\left(\frac{C \nu^{2}}{2}-C_{2}\right) .
\end{aligned}
$$

Here $C$ is the anomaly coefficient and $C_{2}$ is related to the mixed anomaly ${ }^{6}$ in (1.3) by

$$
C_{2}=8 \pi^{2} c_{m}
$$

\footnotetext{
${ }^{5}$ Note that equation (2.5) is valid only if we choose Landau frame. This is the place where the choice of a fluid frame enters our analysis. We are going to use these equations in all our subsequent calculation.

${ }^{6}$ The relation (2.7) was derived in [16]. Here we simply use it.
} 
If we also expand $E, P$ and $q$ in terms of a derivative expansion as $\left.E\right|_{e q}=\bar{E}+\delta E_{(1)}+\delta E_{(2)}+\cdots,\left.\quad P\right|_{e q}=\bar{P}+\delta P_{(1)}+\delta P_{(2)}+\cdots,\left.q\right|_{e q}=\bar{q}+\delta q_{(1)}+\delta q_{(2)}+\cdots$, then from (2.5) it follows that

$$
\delta E_{(1)}=\delta P_{(1)}=\delta q_{(1)}=0 .
$$

Using the fact that $\left(u_{\mu} u^{\mu}=-1\right)$ to all order in derivative expansion we find that

$$
\left[\delta u_{(2)}\right]_{0} \propto\left[\delta u_{(1)}\right]^{i}\left[\delta u_{(1)}\right]_{i}=\text { Parity even } \sim 0 \text { (For our purpose) } .
$$

Also using the Landau gauge condition on the second order stress tensor and current one can show that in equilibrium

$$
\left[T_{(2)}\right]_{00}=\left[T_{(2)}\right]_{0}^{i}=\left[J_{(2)}\right]_{0}=0 .
$$

Note that $\left[T_{(2)}\right]_{00},\left[T_{(2)}\right]_{0}^{i},\left[J_{(2)}\right]_{0}$ are the components of the stress tensor and the charge current which are second order in derivatives obtained from the equation (1.8). While $\left[\Pi^{(2)}\right]_{00},\left[\Pi^{(2)}\right]_{0}^{i},\left[j^{(2)}\right]_{0}$ refer to the components of the stress tensor and the charge current obtained from the equilibrium partition function using (2.3).

Using (2.5), (2.8), (2.9) and (2.10) we get the following form for the second order stress tensor and current evaluated in equilibrium.

$$
\begin{aligned}
{\left[\Pi^{(2)}\right]_{00} } & =\delta E_{(2)} \bar{u}_{0}^{2}+\delta\left(-2 \eta \sigma_{00}\right)+\delta\left(-\zeta \Theta P_{00}\right), \\
{\left[\Pi^{(2)}\right]_{0}^{i} } & =(\bar{E}+\bar{P}) \bar{u}_{0}\left[\delta u_{(2)}\right]^{i}+\delta\left(-2 \eta \sigma_{0}^{i}\right)+\delta\left(-\zeta \Theta P_{0}^{i}\right), \\
{\left[j^{(2)}\right]_{0} } & =\delta q_{(2)} \bar{u}_{0}+\delta\left(\Delta V_{0}\right)+\delta\left(\xi_{l} l_{0}\right)+\delta\left(\xi_{B} B_{0}\right),
\end{aligned}
$$

where $\delta\left(-2 \eta \sigma_{\mu \nu}\right), \delta\left(\zeta \Theta P_{\mu \nu}\right)$ and $\delta\left(\Delta V_{\mu}\right)$ are the two derivative corrections of $\left(-2 \eta \sigma_{\mu \nu}\right)$, $\left(\zeta \Theta P_{\mu \nu}\right)$ and $\left(\Delta V_{\mu}\right)$ when evaluated on $\delta u_{(1)}^{\mu}, \delta T_{(1)}$ and $\delta \nu_{(1)}$.

Now if $Q_{\mu \nu}^{(1)}$ is a tensor which is first order in the derivative expansion satisfying the following two conditions

$$
Q_{\mu \nu}^{(1)} u^{\mu}=0 \quad \text { at all orders and }\left.\quad Q_{\mu \nu}^{(1)}\right|_{\left(\bar{u}^{\mu}, \bar{T}, \bar{\nu}\right)}=0,
$$

then one can show in general that ${ }^{7}$

$$
\delta Q_{00}^{(1)}=\delta\left[Q^{(1)}\right]_{0}^{i}=0 .
$$

Similarly if $Q_{\mu}^{(1)}$ is a vector which is first order in the derivative expansion satisfying

$$
Q_{\mu}^{(1)} u^{\mu}=0 \quad \text { at all orders and }\left.\quad Q_{\mu}^{(1)}\right|_{\left(\bar{u}^{\mu}, \bar{T}, \bar{\nu}\right)}=0,
$$

then it follows that ${ }^{8}$

$$
\delta Q_{0}^{(1)}=0
$$

$$
\begin{aligned}
& { }^{7} 0=\delta\left[u^{\mu} Q_{\mu \nu}^{(1)}\right]=\bar{u}^{\mu} \delta Q_{\mu \nu}^{(1)}+\delta u^{\mu}\left[\left.Q_{\mu \nu}^{(1)}\right|_{(\bar{u} \alpha, \bar{T}, \bar{\nu})}\right]=e^{-\sigma} \delta Q_{0 \nu}^{(1)} . \\
& { }^{8} 0=\delta\left[u^{\mu} Q_{\mu}^{(1)}\right]=\bar{u}^{\mu} \delta Q_{\mu}^{(1)}+\delta u^{\mu}\left[\left.Q_{\mu}^{(1)}\right|_{\left(\bar{u}^{\alpha}, \bar{T}, \bar{\nu}\right)}\right]=e^{-\sigma} \delta Q_{0}^{(1)} .
\end{aligned}
$$


This argument allows us to conclude that

$$
\delta\left(-2 \eta \sigma_{00}\right)=\delta\left(\zeta \Theta P_{00}\right)=\delta\left(-2 \eta \sigma_{0}^{i}\right)=\delta\left(\zeta \Theta P_{0}^{i}\right)=\delta\left(\Delta V_{0}\right)=0 .
$$

Similarly $\delta\left(\xi_{l} l_{\mu}\right)$ and $\delta\left(\xi_{B} B_{\mu}\right)$ are the two derivative corrections of $\left(\xi_{l} l_{\mu}\right)$ and $\left(\xi_{B} B_{\mu}\right)$ when evaluated on $\delta u_{(1)}^{\mu}, \delta T_{(1)}$ and $\delta \nu_{(1)}$. But $l_{\mu}$ and $B_{\mu}$ are already parity odd. Therefore $\delta\left(\xi_{l} l_{\mu}\right)$ and $\delta\left(\xi_{B} B_{\mu}\right)$ are going to be parity even and hence we can set them to zero for our purpose. Putting all this together we have the following result for the components of the stress tensor and the current at the second order in derivative expansion

$$
\begin{aligned}
{\left[\Pi^{(2)}\right]_{00} } & =\delta E_{(2)} \bar{u}_{0}^{2}=e^{2 \sigma} \delta E_{(2)}, \\
{\left[\Pi^{(2)}\right]_{0}^{i} } & =(\bar{E}+\bar{P}) \bar{u}_{0}\left[\delta u_{(2)}\right]^{i}=-e^{\sigma}(\bar{E}+\bar{P})\left[\delta u_{(2)}\right]^{i}, \\
{\left[j^{(2)}\right]_{0} } & =-e^{\sigma} \delta q_{(2)} .
\end{aligned}
$$

Inverting (2.16) and using (2.3) we can determine the second order piece of the equilibrium solution for velocity, temperature and chemical potential in terms of background data. Now the components $\left[\Pi^{(2)}\right]^{i j}$ and $\left[j^{(2)}\right]^{i}$ will give rise to the constraints on the transport coefficients.

$$
\begin{aligned}
{\left[\Pi^{(2)}\right]^{i j} } & =\delta P_{2} g^{i j}-2 \eta \delta \sigma^{i j}-\zeta \delta \Theta g^{i j}+\left[T_{(2)}\right]^{i j}, \\
{\left[j^{(2)}\right]^{i} } & =\bar{q}\left[\delta u_{(2)}\right]^{i}+\Delta \delta\left[V^{i}\right]+\left[J_{(2)}\right]^{i} .
\end{aligned}
$$

We have to use $(2.16)$ to determine $\delta P_{2}$ and $\left[\delta u_{(2)}\right]^{i}$.

The transport coefficients are determined by demanding that (2.17) is satisfied. For convenience let us further split the first equation in (2.17) in two parts, the trace part which is obtained by contracting the equation with $g_{i j}$ and a traceless part which is obtained by subtracting the trace part of the equation from the first equation of (2.17). ${ }^{9}$ These are given by

Trace part :

$\delta P_{2}-\zeta \delta \Theta+\frac{1}{3}\left(-2 \eta \delta \sigma^{i j}+\left[T_{(2)}\right]^{i j}\right) g_{i j}=0$.

Traceless part :

$$
-2 \eta\left(\delta \sigma^{i j}-\frac{g^{i j}}{3}\left(\delta \sigma^{l m} g_{l m}\right)\right)+\left(\left[T_{(2)}\right]^{i j}-\frac{g^{i j}}{3}\left(\left[T_{(2)}\right]^{l m} g_{l m}\right)\right)=0 .
$$

In (2.18) we have used (2.3) to set $\left[\Pi^{(2)}\right]^{i j}$ to zero. Therefore the r.h.s. of (2.18) vanishes.

Transport in the traceless part of the stress tensor. In this subsection we shall analyze the second equation of (2.18).

Traceless part :

$$
-2 \eta\left(\delta \sigma^{i j}-\frac{g^{i j}}{3}\left(\delta \sigma^{l m} g_{l m}\right)\right)+\left(\left[T_{(2)}\right]^{i j}-\frac{g^{i j}}{3}\left(\left[T_{(2)}\right]^{l m} g_{l m}\right)\right)=0 .
$$

We now go through the following steps:

\footnotetext{
${ }^{9}$ Note that here trace is taken with the 3 dimensional metric.
} 
- We first evaluate the second order contribution from $\sigma^{i j}$.

- We then write down the most general form for $\left[T_{(2)}\right]^{\langle\mu \nu\rangle}$ from usual symmetry analysis and on shell independence. In general it will contain many unknown transport transport coefficients to begin with. But if we restrict our attention to only the parity odd ones, the total number of terms is $18\left(\Phi_{i},(i=1, \cdots, 12)\right.$ multiplying 12 independent traceless symmetric tensors and $\chi,(i=1, \cdots, 6)$ multiplying 6 independent scalars, see (1.8)). All these 18 terms have been listed in table 2 .

- One can see that all the $6 \chi_{i}$ s are not going to appear in the combination that we are going to evaluate in (2.19).

- So we have to evaluate the rest of the 12 terms (multiplying $\Phi_{i}$ s on the equilibrium time-independent solution.

For this it is sufficient to substitute only the zeroth order equilibrium solution, since each of these terms already contain two derivatives.

- As mentioned in section 1.1, it turns out that 6 of these twelve terms evaluate to zero in equilibrium. So there are only 6 transport coefficients which can be constrained by this equilibrium analysis. These 6 terms are the following.

$$
\begin{aligned}
& \tau_{\mu \nu}^{(1)}=\bar{\nabla}_{\langle\mu} l_{\nu\rangle}, \quad \tau_{\mu \nu}^{(2)}=\bar{\nabla}_{\langle\mu} B_{\nu\rangle}, \quad \tau_{\mu \nu}^{(3)}=\left(\partial_{\langle\mu} \nu\right) l_{\nu\rangle}, \\
& \tau_{\mu \nu}^{(4)}=\left(\partial_{\langle\mu} \nu\right) B_{\nu\rangle}, \quad \tau_{\mu \nu}^{(5)}=\left(\partial_{\langle\mu} T\right) l_{\nu\rangle}, \quad \tau_{\mu \nu}^{(6)}=\left(\partial_{\langle\mu} T\right) B_{\nu\rangle},
\end{aligned}
$$

where $l^{\mu} \equiv \epsilon^{\mu \nu \alpha \beta} u_{\nu} \partial_{\alpha} u_{\beta}$ and $B^{\mu} \equiv \epsilon^{\mu \nu \alpha \beta} u_{\nu} \partial_{\alpha} \mathcal{A}_{\beta}$. Let us now rewrite the traceless part of the second order fluid stress tensor with these six terms, this is given by

$$
\left[T_{(2)}\right]_{o d d}^{\mu \nu}=\sum_{a=1}^{6} \Phi_{a}\left[\tau^{(a)}\right]^{\mu \nu}+\text { trace part }+ \text { terms that vanish in equilibrium. }
$$

- From (2.19) we see that each of these six $\Phi_{a}$ 's has to be such that, they cancel the contribution of $\delta \sigma_{\mu \nu}$ when all of them are evaluated in equilibrium.

- Evaluating the spatial components of these six terms on the zeroth order equilibrium solution given in (2.5) we obtain

$$
\begin{aligned}
& {\left[\tau^{(1)}\right]^{i j}=\bar{\nabla}^{\langle i} l^{j\rangle}=g^{i l} g^{j m}\left[\frac{\nabla_{l} \bar{l}_{m}+\nabla_{m} \bar{l}_{l}}{2}-\frac{g_{l m}}{3}\left(\nabla_{k} \bar{l}^{k}\right)\right]+\mathcal{O}\left(\partial^{3}\right),} \\
& {\left[\tau^{(2)}\right]^{i j}=\bar{\nabla}^{\langle i} B^{j\rangle}=g^{i l} g^{j m}\left[\frac{\nabla_{l} \bar{B}_{m}+\nabla_{m} \bar{B}_{l}}{2}-\frac{g_{l m}}{3}\left(\nabla_{k} \bar{B}^{k}\right)\right]+\mathcal{O}\left(\partial^{3}\right),} \\
& {\left[\tau^{(3)}\right]^{i j}=\left(\bar{\nabla}^{\langle\mu} \nu\right) l^{\nu\rangle}=g^{i l} g^{j m}\left[\frac{\left(\nabla_{l} \bar{\nu}\right) \bar{l}_{m}+\left(\nabla_{m} \bar{\nu}\right) \bar{l}_{l}}{2}-\frac{g_{l m}}{3}\left(\nabla_{k} \bar{\nu}^{k}\right)\right]+\mathcal{O}\left(\partial^{3}\right),} \\
& {\left[\tau^{(4)}\right]^{i j}=\left(\bar{\nabla}^{\langle\mu} \nu\right) B^{\nu\rangle}=g^{i l} g^{j m}\left[\frac{\left(\nabla_{l} \bar{\nu}\right) \bar{B}_{m}+\left(\nabla_{m} \bar{\nu}\right) \bar{B}_{l}}{2}-\frac{g_{l m}}{3}\left(B^{k} \nabla_{k} \bar{\nu}\right)\right]+\mathcal{O}\left(\partial^{3}\right),} \\
& {\left[\tau^{(5)}\right]^{i j}=\left(\bar{\nabla}^{\langle\mu} T\right) l^{\nu\rangle}=g^{i l} g^{j m}\left[\frac{\left(\nabla_{l} \bar{T}\right) \bar{l}_{m}+\left(\nabla_{m} \bar{T}\right) \bar{l}_{l}}{2}-\frac{g_{l m}}{3}\left(\bar{l}^{k} \nabla_{k} \bar{T}\right)\right]+\mathcal{O}\left(\partial^{3}\right),} \\
& {\left[\tau^{(6)}\right]^{i j}=\left(\bar{\nabla}^{\langle\mu} T\right) B^{\nu\rangle}=g^{i l} g^{j m}\left[\frac{\left(\nabla_{l} \bar{T}\right) \bar{B}_{m}+\left(\nabla_{m} \bar{T}\right) \bar{B}_{l}}{2}-\frac{g_{l m}}{3}\left(\bar{B}^{k} \nabla_{k} \bar{T}\right)\right]+\mathcal{O}\left(\partial^{3}\right),}
\end{aligned}
$$


where $\bar{l}^{i} \equiv-\frac{e^{\sigma}}{2} \epsilon^{i j k} f_{j k}$ and $\bar{B}^{i} \equiv \frac{1}{2} \epsilon^{i j k}\left(F_{j k}+A_{0} f_{j k}\right)$. To evaluate equation (2.22) we have extensively used section 2 of [14].

- The spatial components of the shear tensor gives the following contribution to $\left[\Pi^{(2)}\right]^{i j}$

$$
\begin{gathered}
-2 \eta \delta \sigma^{i j} \\
=-2 \eta\left[\bar{P}^{i \mu} \bar{P}^{j \nu}\left\{\frac{\bar{\nabla}_{\mu}\left[\delta u_{(1)}\right]_{\nu}+\bar{\nabla}_{\nu}\left[\delta u_{(1)}\right]_{\mu}}{2}-\frac{\bar{\nabla}_{\alpha}\left[\delta u_{(1)}\right]^{\alpha}}{3}\right\}\right. \\
\left.+\left(\bar{P}^{j \mu} \delta P^{i \nu}+\bar{P}^{i \nu} \delta P^{j \mu}\right)\left(\frac{\bar{\nabla}_{\mu} \bar{u}_{\nu}+\bar{\nabla}_{\nu} \bar{u}_{\mu}}{2}\right)\right] \\
=-2 \eta\left[g^{i l} g^{j m}\left\{\frac{\nabla_{l}\left[\delta u_{(1)}\right]_{m}+\nabla_{m}\left[\delta u_{(1)}\right]_{l}}{2}-\frac{g_{l m}}{3} \nabla_{k}\left[\delta u_{(1)}\right]^{k}-\frac{g_{l m}}{3}\left(\partial_{k} \sigma\right)\left[\delta u_{(1)}\right]^{k}\right\}\right. \\
\left.+\frac{\delta u^{i} g^{j k}\left(\partial_{k} \sigma\right)+\left[\delta u_{(1)}\right]^{j} g^{i k}\left(\partial_{k} \sigma\right)}{2}\right],
\end{gathered}
$$

where

$$
\bar{P}^{i \mu}=\bar{u}^{i} \bar{u}^{\mu}+G^{i \mu}=G^{i \mu}, \quad \bar{P}^{j \mu} \delta P^{i \nu}=G^{j \mu} \bar{u}^{\nu}\left[\delta u_{(1)}\right]^{i} .
$$

The last term in the last line of (2.23) results from this expansion of the projectors. Substituting (2.5) in (2.23) we obtain the following

$$
\begin{gathered}
-2 \eta \delta \sigma^{i j}=-2 \eta\left[\frac{b_{1}}{2}\left[\tau^{(1)}\right]^{i j}+b_{2}\left[\tau^{(2)}\right]^{i j}+\frac{1}{2}\left(\frac{\partial b_{1}}{\partial \nu}\right)\left[\tau^{(3)}\right]^{i j}+\left(\frac{\partial b_{2}}{\partial \nu}\right)\left[\tau^{(4)}\right]^{i j}\right. \\
\left.+\frac{1}{2}\left(-\frac{b_{1}}{T}+\frac{\partial b_{1}}{\partial T}\right)\left[\tau^{(5)}\right]^{i j}+\left(-\frac{b_{2}}{T}+\frac{\partial b_{2}}{\partial T}\right)\left[\tau^{(6)}\right]^{i j}\right] .
\end{gathered}
$$

Next we have to substitute (2.23) and (2.24) in (2.19). Now to satisfy (2.19) the coefficient of each independent expression should vanish. From examining (2.23) and (2.24) it seems that demanding every independent term to vanish results in more equations than the unknowns which are the transport coefficients. However from the structure of the equations in (2.19) it is clear that the equation admits the following unique solution for the transport coefficients

$$
\begin{array}{ll}
\Phi_{1}=\eta b_{1}, \quad \Phi_{2}=2 \eta b_{2}, & \Phi_{3}=\eta\left(\frac{\partial b_{1}}{\partial \nu}\right), \quad \Phi_{4}=2 \eta\left(\frac{\partial b_{2}}{\partial \nu}\right), \\
\Phi_{5}=\eta\left[-\frac{b_{1}}{T}+\frac{\partial b_{1}}{\partial T}\right], & \Phi_{6}=2 \eta\left[-\frac{b_{2}}{T}+\frac{\partial b_{2}}{\partial T}\right],
\end{array}
$$

where

$$
b_{1}=\frac{T^{3}}{E+P}\left(\frac{2 C \nu^{3}}{3}-4 C_{2} \nu\right), \quad b_{2}=\frac{T^{2}}{E+P}\left(\frac{C \nu^{2}}{2}-C_{2}\right)
$$

and $E, P, T$ is the energy density, pressure and temperature respectively. $\nu=\frac{\mu}{T}$ refers to the chemical potential, and $\eta$ is the shear viscosity. $C$ is the gauge anomaly coefficient, while $C_{2}$ is related to the mixed anomaly by (1.11). The transport coefficient $\Phi_{1}$ gives rise to chiral dispersion relations in the shear mode [13]. 
Transport in the trace of the stress tensor and current. We will now constrain the transport coefficients that occur in the trace part of the stress tensor and the current using the equilibrium partition function. We will first rewrite the relevant part of the current and the trace part of the stress tensor up to 2nd order in derivative expansion. Using (2.3) and (2.17) we obtain the following equation for the trace part of the stress tensor and current at second order.

$$
\text { Trace part : } \begin{aligned}
0 & =\delta P_{2}-\zeta \delta \Theta+\frac{1}{3}\left(-2 \eta \delta \sigma^{i j}+\left[T_{(2)}\right]^{i j}\right) g_{i j} \\
& =\delta P_{2}-\zeta \delta \Theta+\left[\sum_{1}^{6} \chi_{i} \mathcal{S}_{i}\right]_{e q} \\
& =\left[-\zeta \delta \Theta+\left(\frac{\partial P}{\partial E}\right)_{q} \delta E_{(2)}+\left(\frac{\partial P}{\partial q}\right)_{E} \delta q_{(2)}\right]+\left[\sum_{1}^{6} \chi_{i} \mathcal{S}_{i}\right]_{e q}
\end{aligned}
$$

Current : $\left[J_{(2)}\right]^{i}=\left[j^{(2)}\right]^{i}-\delta\left[\Delta V^{i}\right]-q\left[\delta u_{(2)}\right]^{i}$

$$
\Rightarrow\left[\sum_{k=1}^{9} \Delta_{k} \mathcal{V}_{(k)}^{i}\right]_{e q}=2 \bar{T}\left(\frac{\partial M_{1}}{\partial \bar{T}}\right) \epsilon^{i j k}\left(\partial_{j} \bar{T}\right)\left(\partial_{k} \bar{\nu}\right)-\delta\left[\Delta V^{i}\right]-q\left[\delta u_{(2)}\right]^{i} .
$$

Both in (2.27) and (2.28) we have used (1.8) to write the fluid stress tensor and the current in terms of the transport coefficients. Also in (2.27) to evaluate the combination $\left(-2 \eta \delta \sigma^{i j}+\left[T_{(2)}\right]^{i j}\right) g_{i j}$ we have used (2.24) and (2.25). One can see that the transport coefficients $\Phi_{i}$ s drop out.

Using (2.16) and (2.3) we evaluate $\delta E_{2}, \delta q_{2}$ and $\left[\delta u_{(2)}\right]^{i}$, this results in

$$
\begin{gathered}
\delta E_{2}=e^{-2 \sigma} \Pi_{00}^{(2)}=2 \bar{T}^{2}\left(\frac{\partial M_{1}}{\partial \bar{T}}\right)\left(\bar{B}^{i} \partial_{i} \bar{\nu}\right)-2 \bar{T}^{3}\left(\frac{\partial M_{2}}{\partial \bar{T}}-\bar{\nu} \frac{\partial M_{1}}{\partial \bar{T}}\right)\left(\bar{l}^{i} \partial_{i} \bar{\nu}\right), \\
\delta q_{2}=e^{-\sigma} j_{0}^{(2)}=2\left(\frac{\partial M_{1}}{\partial \bar{T}}\right)\left(\bar{B}^{i} \partial_{i} \bar{T}\right)-2 \bar{T}\left(\frac{\partial M_{2}}{\partial \bar{T}}-\bar{\nu} \frac{\partial M_{1}}{\partial \bar{T}}\right)\left(\bar{l}^{i} \partial_{i} \bar{T}\right), \\
{\left[\delta u_{(2)}\right]^{i}=-\left(\frac{e^{-\sigma}}{\bar{E}+\bar{P}}\right)\left[\Pi^{(2)}\right]_{0}^{i}=-\left(\frac{2 \bar{T}^{2}}{E+P}\right)\left(\frac{\partial M_{2}}{\partial \bar{T}}-\bar{\nu} \frac{\partial M_{1}}{\partial \bar{T}}\right) \epsilon^{i j k}\left(\partial_{j} \bar{T}\right)\left(\partial_{k} \bar{\nu}\right) .}
\end{gathered}
$$

Now we have to compute $\delta \Theta$ and $\delta\left(\Delta V^{i}\right)$.

$$
\begin{aligned}
\delta \Theta= & \bar{\nabla}_{\mu}\left[\delta u_{(1)}\right]^{\mu}=\nabla_{k}\left[\delta u_{(1)}\right]^{k}-\left(\frac{\partial_{k} \bar{T}}{\bar{T}}\right)\left[\delta u_{(1)}\right]^{k} \\
= & \frac{1}{2}\left(\frac{\partial b_{1}}{\partial \bar{T}}-\frac{2 b_{1}}{\bar{T}}\right)\left(\bar{l}^{i} \partial_{i} \bar{T}\right)+\frac{1}{2}\left(\frac{\partial b_{1}}{\partial \bar{\nu}}-2 b_{2} \bar{T}\right)\left(\bar{l}^{i} \partial_{i} \bar{\nu}\right) \\
& +\left(\frac{\partial b_{2}}{\partial \bar{T}}-\frac{b_{2}}{\bar{T}}\right)\left(\bar{B}^{i} \partial_{i} \bar{T}\right)+\left(\frac{\partial b_{2}}{\partial \bar{\nu}}\right)\left(\bar{B}^{i} \partial_{i} \bar{\nu}\right) .
\end{aligned}
$$

To derive (2.30) we have used the following identities

$$
\nabla_{i} \bar{l}^{i}=-\left(\frac{\partial_{i} \bar{T}}{\bar{T}}\right) \bar{l}^{i}, \quad \nabla_{i} \bar{B}^{i}=-\bar{T} \bar{l}^{i} \partial_{i} \bar{\nu}
$$


Similarly $\delta V^{i}$ is given by the following expression

$$
\delta\left\{\Delta V^{i}\right\}=\Delta G^{i \mu} \mathcal{F}_{\mu \nu} \delta u^{\nu}=\Delta \epsilon^{i j k}\left[\delta u_{(1)}\right]_{j} \bar{B}_{k}=\Delta\left(\frac{b_{1}}{2}\right) \epsilon^{i j k} \bar{l}_{j} \bar{B}_{k},
$$

where $\mathcal{F}_{\mu \nu}=\partial_{\mu} \mathcal{A}_{\nu}-\partial_{\nu} \mathcal{A}_{\mu}$.

Now we have to evaluate the 6 scalars $\left(\mathcal{S}_{i}, \quad i=1, \cdots, 6\right)$ and the $i$ th component of the 9 vectors $\left(\mathcal{V}_{(k)}^{i}, \quad k=1, \cdots, 9\right)$ on the equilibrium solution. We need the equilibrium solution only at zeroth order since all of them already contain two derivatives. Explicit expressions for all these terms are listed in table 2 .

As mentioned in section 1.1, only 6 terms, 4 scalars and 2 vectors are non-vanishing on the zeroth order equilibrium solution. Thus the relevant parts of the second order current and trace of the stress tensor are given by

$$
\begin{aligned}
{\left.\left[T_{(2)}\right]^{\alpha \beta}\right|_{\text {trace part }} } & =P^{\alpha \beta}\left[\chi_{1}\left(l^{\mu} \partial_{\mu} \nu\right)+\chi_{2}\left(B^{\mu} \partial_{\mu} \nu\right)+\chi_{3}\left(l^{\mu} \partial_{\mu} T\right)+\chi_{4}\left(B^{\mu} \partial_{\mu} T\right)\right], \\
J_{(2)}^{\mu} & =\Delta_{1}\left[\epsilon^{\mu \nu \alpha \beta} u_{\nu}\left(\partial_{\alpha} \nu\right)\left(\partial_{\beta} T\right)\right]+\Delta_{2}\left[\epsilon^{\mu \nu \alpha \beta} u_{\nu} B_{\alpha} l_{\beta}\right] .
\end{aligned}
$$

We then evaluate all these six terms on the zeroth order equilibrium solution.

$$
\begin{aligned}
\left(l^{\mu} \partial_{\mu} \nu\right) & =\left(\bar{l}^{i} \partial_{i} \bar{\nu}\right), \\
\left(B^{\mu} \partial_{\mu} \nu\right) & =\left(\bar{B}^{i} \partial_{i} \bar{\nu}\right), \\
\left(l^{\mu} \partial_{\mu} T\right) & =\left(\bar{l}^{i} \partial_{i} \bar{T}\right), \\
\left(B^{\mu} \partial_{\mu} T\right) & =\left(\bar{B}^{i} \partial_{i} \bar{T}\right), \\
\epsilon^{i \nu \alpha \beta} u_{\nu}\left(\partial_{\alpha} \nu\right)\left(\partial_{\beta} T\right) & =\epsilon^{i j k}\left(\partial_{j} \bar{\nu}\right)\left(\partial_{k} \bar{T}\right), \\
\epsilon^{i \nu \alpha \beta} u_{\nu} B_{\alpha} l_{\beta} & =\epsilon^{i j k} \bar{B}_{j} \bar{l}_{k} .
\end{aligned}
$$

Substituting (2.34) in (2.33) we express the l.h.s. of (2.33) in terms of the background data and the two arbitrary functions $M_{1}$ and $M_{2}$ of the second order parity odd partition function. Now using (2.27) and (2.3) we can express the 5 transport coeffcients appearing in (2.33) in terms of $M_{1}$ and $M_{2}$. Since one cannot generate a term of the form $\epsilon^{i j k} l_{j} B_{k}$ from the partition function, the 6th transport coefficients $\Delta_{2}$ will be completely determined by the correction of the first order current. This is similar to the way the transport coefficients $\Phi_{i}$ 's, which appear in the traceless part of the stress tensor were determined. The end result of this step is

$$
\begin{aligned}
& \Delta_{2}=-\frac{\Delta b_{1}}{2} \\
& \chi_{1}=-2 R_{1} \bar{T}^{3}\left[\bar{\nu} \frac{\partial M_{1}}{\partial \bar{T}}-\frac{\partial M_{2}}{\partial \bar{T}}\right]+\zeta\left(\frac{1}{2} \frac{\partial b_{1}}{\partial \nu}-b_{2} \bar{T}\right), \\
& \chi_{2}=-2 R_{1} \bar{T}^{2}\left(\frac{\partial M_{1}}{\partial \bar{T}}\right)+\zeta\left(\frac{\partial b_{2}}{\partial \nu}\right), \\
& \chi_{3}=-2 R_{2} \bar{T}\left[\bar{\nu} \frac{\partial M_{1}}{\partial \bar{T}}-\frac{\partial M_{2}}{\partial \bar{T}}\right]+\frac{\zeta}{2}\left(\frac{\partial b_{1}}{\partial \bar{T}}-\frac{2 b_{1}}{\bar{T}}\right), \\
& \chi_{4}=2 R_{2}\left(\frac{\partial M_{1}}{\partial \bar{T}}\right)+\zeta\left(\frac{\partial b_{2}}{\partial \bar{T}}-\frac{b_{2}}{\bar{T}}\right), \\
& \Delta_{1}=2 \bar{T}\left(\frac{\partial M_{1}}{\partial \bar{T}}\right)+\left(\frac{2 q T^{2}}{E+P}\right)\left(\frac{\partial M_{2}}{\partial \bar{T}}-\bar{\nu} \frac{\partial M_{1}}{\partial \bar{T}}\right),
\end{aligned}
$$


where

$$
\begin{aligned}
R_{1} & =\left(\frac{\partial P}{\partial E}\right)_{q}, & R_{2} & =\left(\frac{\partial P}{\partial q}\right)_{E} \\
b_{1} & =\frac{T^{3}}{E+P}\left(\frac{2 C \nu^{3}}{3}-4 C_{2} \nu\right), & b_{2} & =\frac{T^{2}}{E+P}\left(\frac{C \nu^{2}}{2}-C_{2}\right) .
\end{aligned}
$$

Eliminating $M_{1}$ and $M_{2}$ from these expressions we get three relations among the remaining 5 second order transport coefficients.

$$
\begin{aligned}
\Delta_{2} & =-\frac{\Delta b_{1}}{2}, \\
T^{2} R_{1}\left[\chi_{3}-\frac{\zeta}{2}\left(\frac{\partial b_{1}}{\partial T}-\frac{2 b_{1}}{T}\right)\right]-R_{2}\left[\chi_{1}-\frac{\zeta}{2}\left(\frac{\partial b_{1}}{\partial \nu}-2 b_{2} T\right)\right] & =0, \\
T^{2} R_{1}\left[\chi_{4}-\zeta\left(\frac{\partial b_{2}}{\partial T}-\frac{b_{2}}{T}\right)\right]+R_{2}\left[\chi_{2}-\zeta\left(\frac{\partial b_{2}}{\partial \nu}\right)\right] & =0, \\
R_{1} T \Delta_{1}+\left[\chi_{2}-\zeta\left(\frac{\partial b_{2}}{\partial \nu}\right)\right]-\frac{q}{(E+P)}\left[\chi_{1}-\frac{\zeta}{2}\left(\frac{\partial b_{1}}{\partial \nu}-2 b_{2} T\right)\right] & =0 .
\end{aligned}
$$

\section{Kubo formula for the transport coefficients $\Phi_{1}, \Phi_{2}$}

In this section we derive the relations obtained for the transport coefficients $\Phi_{1}$ and $\Phi_{2}$ given in (2.25) using the Kubo formula. We consider the following equilibrium background for the metric, gauge field and the velocity

$$
g_{\mu \nu}^{(0)}=\operatorname{diag}(-1,1,1,1), \quad \mathcal{A}_{\mu}=\left(\nu^{(0)} T_{0}, 0,0,0\right), \quad u^{\mu}=(1,0,0,0) .
$$

The chemical potential $\nu^{(0)}$ and the temperature $T_{0}$ are constants and do not depend on space-time. Since the energy $E^{(0)}$ and the pressure $P^{(0)}$ can be thought of as functions of the temperature and the chemical potential, they are also constants in space-time. Now consider the following non-zero metric perturbations about this background

$$
\delta g_{t x}=h_{t x}, \quad \delta g_{t z}=h_{t z}, \quad \delta g_{y x}=h_{y x}, \quad \delta g_{y z}=h_{y z} .
$$

The gauge field perturbations are given by

$$
\delta \mathcal{A}_{\mu}=\left(0, a_{x}, 0, a_{z}\right) .
$$

The fluid velocity is close to the rest frame and its perturbations are given by

$$
\delta u_{\mu}=\left(0, v^{x}, 0, v^{z}\right) .
$$

All perturbations are assumed to have dependence only in the time $t$ and $y$-direction. In appendix B we will show that the background and the perturbations considered in equations (3.1) to (3.4) consistently solve the linearized fluid equations without the need for turning on any other perturbations. A simple reason that these perturbations consistently solve the linearized fluid equations is that they are all in the spin-2 shear sector and therefore they decouple from the rest.

To derive Kubo formulae for transport coefficients, we consider the constitutive relations for the stress tensor and the charge current as one point functions in the presence of external sources. We then obtain two point functions for the currents by differentiating 
with respect to the metric and the gauge field perturbations. Working this out to the linear order in perturbations will result in Kubo formulae for the transport coefficients $\Phi_{1}, \Phi_{2}$.

To proceed we will require the the Christoffel symbols to the linear order in perturbations. The non-vanishing elements at the linear order are given by

$$
\begin{array}{rlrl}
\Gamma_{x y}^{t} & =-\frac{1}{2}\left(\partial_{y} h_{t x}-\partial_{t} h_{y x}\right), & \Gamma_{z y}^{t}=-\frac{1}{2}\left(\partial_{y} h_{t z}-\partial_{t} h_{z y}\right), \\
\Gamma_{t t}^{x}=\partial_{t} h_{t x}, & \Gamma_{t y}^{x}=\frac{1}{2}\left(\partial_{y} h_{t x}+\partial_{t} h_{y x}\right), & \Gamma_{y y}^{x}=\partial_{y} h_{x y}, \\
\Gamma_{t t}^{z}=\partial_{t} h_{z 0}, & \Gamma_{t y}^{z}=\frac{1}{2}\left(\partial_{y} h_{t z}+\partial_{t} h_{y z}\right), & \Gamma_{y y}^{x}=\partial_{y} h_{z y}, \\
\Gamma_{t x}^{y}=\frac{1}{2}\left(\partial_{t} h_{y x}-\partial_{y} h_{t x}\right), & \Gamma_{t z}^{y}=\frac{1}{2}\left(\partial_{t} h_{y z}-\partial_{y} h_{t z}\right) .
\end{array}
$$

Evaluating the inverse metric to the linear order we obtain

$$
h^{t x}=h_{t x}, \quad h^{t z}=h_{t z}, \quad h^{y x}=-h_{y x}, \quad h^{y z}=-h_{y z} .
$$

The covariant components of the velocity are given by

$$
u_{\mu}=\left(-1, v^{x}+h_{x t}, 0, v^{z}+h_{z t}\right) .
$$

We now evaluate various components of the stress tensor to the linear order in the perturbations. From the list of terms that contribute at 2 nd order in derivatives given in table 2 we see that in the background we have chosen all the scalars $\mathcal{S}_{i}$ vanish. The reason for this is for the background all the thermodynamic functions are independent of space-time. We now examine the traceless part of the stress tensor. Note that the contributions from $\tau_{\mu \nu}^{(i)}$ for $i=3,4,5,6,7,8,9,10,11$ vanish since the thermodynamic functions are independent of space-time. What remains to be evaluated are the contributions from $\tau_{\mu}^{(i)}$ for $i=1,2$ and $i=12$. Let us first examine the $t x$ and the $t y$ component of the stress tensor. To the second order in derivatives and to the linear order in the perturbation this is given by

$$
\begin{aligned}
& T^{t x}=\left(E^{(0)}+P^{(0}\right) v^{x}+P^{(0)} h_{t x}, \\
& T^{t z}=\left(E^{(0)}+P^{(0)}\right) v^{z}+P^{(0)} h_{t z}, \\
& T^{t y}=0 .
\end{aligned}
$$

Note that $\sigma^{t x}, \sigma^{t z},\left[\tau^{(i)}\right]^{t x}$ and $\left[\tau^{(i)}\right]^{t z}$ for $i=1,2,12$ do not contribute at the linear order. The reason is that the term $\nabla_{\alpha} u_{\beta}$ and $\nabla_{\alpha} l_{\beta}$ is a first order term, therefore one has to evaluate the projector for these components say $P^{t \alpha} P^{x \beta}$ at the zeroth order, which vanishes.

Lets examine the $y x$ component of the stress tensor. We need to evaluate the contributions from $\left[\tau^{(i)}\right]^{y x}$ for $i=1,2,12$. These are given by ${ }^{10}$

$$
\begin{aligned}
{\left[\tau^{(1)}\right]^{y x} } & =\frac{1}{2} \partial_{y}^{2}\left(v^{z}+h_{z t}\right), \\
{\left[\tau^{(2)}\right]^{y x} } & =\frac{1}{2} \partial_{y}^{2} a_{z}, \\
{\left[\tau^{(12)}\right]^{y x} } & =\frac{1}{2} \partial_{y}\left(\partial_{y} v^{z}+\partial_{t} h_{y z}\right) .
\end{aligned}
$$

\footnotetext{
${ }^{10}$ In evaluating these contributions we take $\epsilon^{0123}=\frac{1}{\sqrt{-g}}$.
} 
We also need the contribution of the shear tensor to the linear order. This is given by

$$
\sigma^{y x}=\frac{1}{2}\left(\partial_{y} v^{x}+\partial_{t} h_{y x}\right)
$$

Considering all these contributions along with the contribution to the stress tensor to the zeroth order in derivative we obtain

$$
\begin{aligned}
T^{y x}= & -P^{(0)} h_{y x}-\eta\left(\partial_{y} v^{x}+\partial_{t} h_{y x}\right) \\
& +\frac{1}{2} \Phi_{1} \partial_{y}^{2}\left(v^{z}+h_{z t}\right)+\frac{1}{2} \Phi_{2} \partial_{y}^{2} a_{z} \\
& +\frac{\Phi_{12}}{2} \partial_{y}\left(\partial_{y} v^{z}+\partial_{t} h_{y z}\right) .
\end{aligned}
$$

The equations of motion for the $x$ component of the stress tensor to the linear order in the fields is given by

$$
\partial_{t} T^{t x}+\partial_{t} h_{t x} T^{t t}+\partial_{y} T^{y x}=0 .
$$

Here $T^{t t}$ is the zeroth order $t t$ component of the stress tensor which is given by

$$
T^{t t}=E^{(0)} .
$$

Fourier transforming these equations and taking the zero frequency limit or taking the time independent situation we obtain the Ward identities for the one point function of the stress tensor.

$$
\lim _{\omega \rightarrow 0} T^{y x}(k)=0 .
$$

We can now differentiate these with respect to the background fields $h_{z t}$, and $a_{z}$ and obtain Kubo formulae for the transport coefficients $\Phi_{1}$ and $\Phi_{2}$ respectively. A similar procedure for the $y z$ component of the stress tensor yields the same result.

To proceed we first eliminate $v^{x}$ and $v^{z}$ using (3.8). This results in the following equations

$$
\begin{aligned}
T^{y x}= & -P^{(0)} h_{y x}-\eta\left(\frac{\partial_{y} T^{t x}-P^{(0)} \partial_{y} h_{t x}}{E^{(0)}+P^{(0}}\right) \\
& +\frac{\Phi_{1}}{2}\left(\frac{\partial_{y}^{2} T^{t z}+E^{(0)} \partial_{y}^{2} h_{t z}}{E^{(0)}+P^{(0)}}\right)+\frac{\Phi_{2}}{2} \partial_{y}^{2} a_{z} \\
& +\frac{\Phi_{12}}{2}\left(\frac{\partial_{y}^{2} T^{t z}-P^{(0)} \partial_{y}^{2} h_{t z}}{E^{(0)}+P^{(0}}\right) .
\end{aligned}
$$

Here we have already used time-independence to drop the time derivatives. Fourier transforming these equations we obtain

$$
\begin{aligned}
T^{y x}(k)= & -P^{(0)} h_{y x}-\eta\left(\frac{i k T^{t x}-i k P^{(0)} h_{t x}}{E^{(0)}+P^{(0}}\right) \\
& -\frac{\Phi_{1}}{2}\left(\frac{k^{2} T^{t z}+E^{(0)} k^{2} h_{t z}}{E^{(0)}+P^{(0)}}\right)-\frac{\Phi_{2}}{2} k^{2} a_{z} \\
& -\frac{\Phi_{12}}{2} k^{2}\left(\frac{T^{t z}-P^{(0)} h_{t z}}{E^{(0)}+P^{(0)}}\right) \\
= & 0 .
\end{aligned}
$$


The last equality in the above equation implements the Ward identity given in (3.14) and $k$ is the momentum in the $y$ direction. Let us now focus on the expression for $T^{y x}$ a similar analysis can be repeated for $T^{y z}$. Differentiating the Ward identity for $T^{y x}$ with respect to $h_{z t}$ and $a_{z}$ and setting the other backgrounds to zero we obtain the following two equations

$$
\begin{gathered}
\frac{k^{2}}{E^{(0)}+P^{(0)}}\left[\left(\Phi_{1}\left\langle T^{t z}(k) T^{t z}(-k)\right\rangle+E^{(0)}\right)+\Phi_{12}\left(\left\langle T^{t z}(k) T^{t z}(-k)\right\rangle-P^{(0)}\right)\right] \\
=-i k \frac{2 \eta}{E^{(0)}+P^{(0)}}\left\langle T^{t x}(k) T^{t z}(-k)\right\rangle, \\
\frac{\Phi_{1}+\Phi_{12}}{E^{(0)}+P^{(0)}} k^{2}\left\langle T^{t z}(k) j^{z}(-k)\right\rangle+\Phi_{2} k^{2}=-i k \frac{2 \eta}{E^{(0)}+P^{(0)}}\left\langle T^{t z}(k) j^{z}(-k)\right\rangle .
\end{gathered}
$$

To obtain the first equation we have differentiated with respect to $h_{t z}$ and set all the other backgrounds to zero. The second equation is obtained by differentiating the Ward identity with respect to $a_{z}$ and setting the remaining backgrounds to zero. The equations in (3.17) are sufficient to determine the transport coefficients $\Phi_{1}, \Phi_{2}$. From [9] we have the following results for the various two point functions.

$$
\begin{aligned}
\lim _{k \rightarrow 0, \omega \rightarrow 0}\left\langle T^{t x}(k) T^{t z}(-k)\right\rangle & =i k\left(\frac{C}{3}\left(\nu^{(0)} T^{(0)}\right)^{3}-2 C_{2}\left(T^{(0)}\right)^{3} \nu^{(0)}\right), \\
\lim _{k \rightarrow 0, \omega \rightarrow 0}\left\langle T^{t x}(k) j^{z}(-k)\right\rangle & =i k\left(\frac{C}{2}\left(\nu^{(0)} T^{(0)}\right)^{2}-C_{2}\left(T^{(0)}\right)^{2}\right) .
\end{aligned}
$$

These results are given in equations (79)-(81) for a system with 3 chemical potentials and equations (123)-(125) for a system with a single chemical potential of [9]. The definition of the variables for the two point functions used is given in equation (47). This reference also uses the normalization

$$
\frac{-C}{8}=\frac{1}{32 \pi^{2}}, \quad \frac{c_{m}}{4}=\frac{1}{768 \pi^{2}}, \quad C_{2}=\frac{1}{24},
$$

for the gauge anomaly and we have rewritten the chemical potential $\mu$ in terms of the variable $\nu$. From [17] we can read out the following correlators

$$
\lim _{k \rightarrow 0, \omega \rightarrow 0}\left\langle T^{t z}(k) T^{t z}(-k)\right\rangle=P^{(0)}, \quad \lim _{k \rightarrow 0, \omega \rightarrow 0}\left\langle T^{t z}(k) j^{z}(-k)\right\rangle=0 .
$$

These correlators are mentioned below equation (2.16) of reference [17]. Substituting the formulae for the two point functions given in (3.18) and (3.20) into the equations given in (3.17) we obtain

$$
\begin{aligned}
\Phi_{1} & =\frac{2 \eta}{E^{(0)}+P^{(0)}}\left(\frac{C}{3}\left(\nu^{(0)} T^{(0)}\right)^{3}-2 C_{2}\left(T^{(0)}\right)^{3} \nu^{(0)}\right), \\
\Phi_{2} & =\frac{2 \eta}{E^{(0)}+P^{(0)}}\left(\frac{C}{2}\left(\nu^{(0)} T^{(0)}\right)^{2}-C_{2}\left(T^{(0)}\right)^{2}\right) .
\end{aligned}
$$

These expressions agree with that derived using the equilibrium partition function method which are given in (2.25). 


\section{Chiral shear waves}

In this section we examine the effects of the second order parity transport coefficients on linearized dispersion relations about the equilibrium characterized by the following background given in (3.1). Note that the temperature $T_{0}$ and the chemical potential $\nu^{(0)}$ are independent of space-time and therefore all other thermodynamic variables are constants in space-time. We consider shear modes ${ }^{11}$ for this we examine velocity perturbations of the form

$$
\delta u^{\mu}=\left(0, v^{x}, 0, v^{z}\right) .
$$

These perturbations depend only on time $t$ and the $y$-direction. We include all the terms in the stress tensor to the 2 nd order in derivatives given by

$$
T^{\mu \nu}=\left[T_{(0)}\right]^{\mu \nu}+\left[T_{(1)}\right]^{\mu \nu}+\left[T_{(2)}\right]_{o d d}^{\mu \nu},
$$

where $\left[T_{(0)}\right]^{\mu \nu}$ and $\left[T_{(1)}\right]^{\mu \nu}$ are given in (1.4) and $\left[T_{(2)}\right]_{o d d}^{\mu \nu}$ is given in (1.8). We now write down the contribution to the stress tensor from these velocity fluctuations which are linear order in the fluctuations. It can be seen that the only contributions from 2 nd order which arise are from the term involving $\Phi_{1}$ and $\Phi_{12}$. Thus the stress tensor to the linear order in velocity fluctuations is given by

$$
\begin{aligned}
& \delta T^{t x}=\left(E^{(0)}+P^{(0)}\right) v^{x}, \quad \delta T^{t z}=\left(E^{(0)}+P^{(0)}\right) v^{z}, \\
& \delta T^{y x}=-\eta \partial_{y} v^{x}+\frac{\Phi_{1}}{2} \partial_{y}^{2} v^{z}+\frac{\Phi_{12}}{2} \partial_{y}^{2} v^{z} \\
& \delta T^{y z}=-\eta \partial_{y} v^{z}-\frac{\Phi_{1}}{2} \partial_{y}^{2} v^{x}-\frac{\Phi_{12}}{2} \partial_{y}^{2} v^{x} .
\end{aligned}
$$

We now substitute these values in the equations of motion for the stress tensor given by

$$
\begin{aligned}
\partial_{t} \delta T^{t x}+\partial_{y} T^{y x} & =0, \\
\partial_{t} \delta T^{t z}+\partial_{y} T^{y z} & =0 .
\end{aligned}
$$

Substituting the expressions for the stress tensor from (4.3) into the above equations and then taking the Fourier transform of these equations result in the following set of coupled equations

$$
\begin{aligned}
& \left(-i \omega\left(E^{(0)}+P^{(0)}\right)+\eta k^{2}\right) v^{x}-i \frac{k^{3}}{2}\left(\Phi_{1}+\Phi_{12}\right) v^{z}=0, \\
& \left(-i \omega\left(E^{(0)}+P^{(0)}\right)+\eta k^{2}\right) v^{z}+i \frac{k^{3}}{2}\left(\Phi_{1}+\Phi_{12}\right) v^{x}=0,
\end{aligned}
$$

From these equations we see that the two shear modes split depending on their chirality. The dispersion relations for these modes are given by

$$
\omega=-i \frac{\eta}{E^{(0)}+P^{(0)}} k^{2} \mp \frac{i}{2\left(E^{(0)}+P^{(0)}\right)}\left(\Phi_{1}+\Phi_{12}\right) k^{3} .
$$

\footnotetext{
${ }^{11}$ We have shown that none of the parity odd transport coefficients modify the sound or the charge dissipation mode for the equilibrium characterized by (3.1).
} 
Thus in the basis we have used to list the second order transport coefficients both $\Phi_{1}$ as well as $\Phi_{12}$ contribute to the splitting of the shear modes. Earlier studies of the chiral shear modes were restricted to the conformal transport at second order, therefore the contribution of $\Phi_{12}$ to the splitting was not noticed. Let us call the coefficient of $k^{3}$ as the chiral dispersion coefficient and define

$$
D=\frac{1}{2\left(E^{(0)}+P^{(0)}\right)}\left(\Phi_{1}+\Phi_{12}\right)
$$

Using holography we now show that for the case $\mathcal{N}=4$ Yang-Mills, $\Phi_{12}=0$. We will also check the relation of $\Phi_{1}$ to the anomaly coefficient derived in this paper using the holographic result for this transport coefficient. The holographic dual of this system is given by the Reisner Nordström black hole in $A d S_{5}$. We will use the notations of [2]. The five dimensional action we consider is given by

$$
S=\frac{1}{16 \pi G_{5}} \int \sqrt{-g_{5}}\left(R+12-F_{A B} F^{A B}-\frac{4 \kappa}{3} \epsilon^{L A B C D} A_{L} F_{A B} F_{C D}\right)
$$

The equations of motion of the above action are

$$
\begin{aligned}
G_{A B}-6 g_{A B}+2\left(F_{A C} F_{B}^{C}+\frac{1}{4} g_{A B} F_{C D} F^{C D}\right) & =0 \\
\nabla_{B} F^{A B}+\kappa \epsilon^{A B C D E} F_{B C} F_{D E} & =0
\end{aligned}
$$

where $G_{A B}$ is the five dimensional Einstein tensor. The Reisner-Nordström black brane solution in Eddington Finkelstein coordinates is given by

$$
\begin{aligned}
d s^{2} & =-2 u_{\mu} d x^{\mu} d r-r^{2} V(r, m, q) u_{\mu} u_{\nu} d x^{\mu} d x^{\nu}+r^{2} P_{\mu \nu} d x^{\mu} d x^{\nu} \\
A & =\frac{\sqrt{3} q}{2 r^{2}} u_{\mu} d x^{\mu}
\end{aligned}
$$

and

$$
u_{\mu} d x^{\mu}=d v, \quad V(r, m, q)=1-\frac{m}{r^{4}}+\frac{q^{2}}{r^{6}} .
$$

Let $R$ be the radius of the outer horizon of the black hole. We then define the quantities

$$
M \equiv \frac{m}{R^{4}}, \quad Q=\frac{q}{R^{3}}, \quad Q^{2}=M-1 .
$$

The last equation results from the fact that $R$ is the largest root of $V(r)=0$. The thermodynamic quantities of this black hole are given by

$$
T=\frac{R}{2 \pi}\left(2-Q^{2}\right), \quad \mu=2 \sqrt{3} Q R,
$$

where $T$ is the Hawking temperature and $Q$ the charge density. The energy density, pressure and the shear viscosity in terms of these variables are given by

$$
E^{(0)}=\frac{3 M R^{4}}{16 \pi G_{5}}, \quad P^{(0)}=\frac{M R^{4}}{16 \pi G_{5}} \quad \eta=\frac{R^{3}}{16 \pi G_{5}}=\frac{s}{4 \pi} .
$$


Before we proceed we first relate the anomaly coefficient $C$ to the Chern-Simons coefficient $\kappa$. The boundary current is given by

$$
J^{\mu}=\left.\frac{1}{16 \pi G_{5}} \sqrt{-g_{5}} F^{r \mu}\right|_{r \rightarrow \infty},
$$

where $r$ is the radial direction. Here we are using the definition of the current which is consistent with the Page charge using which the charge density of the black hole is evaluated. There are other definitions of current as discussed in footnote 3 of [2]. The bulk equations of motion for the gauge field results in the following conservation law for the current.

$$
\partial_{\mu} J^{\mu}=-\frac{\kappa}{16 \pi G_{5}} \epsilon^{\mu \nu \rho \sigma} F_{\mu \nu} F_{\rho \sigma} .
$$

We now have to identify the relation between the gauge field used in field theory and that of the bulk gauge field. Note that the chemical potential value is related to the horizon value of the gauge field. Comparing the horizon value of the gauge field in (4.10) and (4.13) we see that the relation between the bulk gauge field and the gauge field is given by

$$
A_{\mu}^{\text {field }}=4 A_{\mu}^{\text {bulk }}
$$

The relation is important since we have already used this normalization to define the thermodynamics of the boundary gauge theory. The gauge fields in the field theory must be defined consistent with this thermodynamics. Substituting the relation (4.17) into the conservation law (4.16) we obtain

$$
\partial_{\mu} J^{\mu}=-\frac{\kappa}{256 \pi G_{5}} \epsilon^{\mu \nu \rho \sigma} F_{\mu \nu}^{\text {field }} F_{\rho \sigma}^{\text {field }} .
$$

Now all quantities are defined in the field theory. Comparing with the current conservation law in (1.3) we obtain

$$
C=\frac{\kappa}{32 \pi G_{5}} .
$$

The transport coefficient $\frac{\Phi_{1}}{2}$ can be identified to the coefficient $\mathcal{N}_{7}$ in the notation of [2], equation (4.37) see also $[3,18]$. Reading out the holographic value of $\mathcal{N}_{7}$ from equation (4.38) of [2] we have

$$
\frac{\Phi_{1}}{2}=\frac{1}{16 \pi G_{5}} \frac{\sqrt{3}}{M}(M-1)^{\frac{3}{2}} R^{2} \kappa
$$

Note that the action given in (4.8) captures the situation when the gravitational anomaly is zero. Using the relations in $(4.12),(4.13)$ and (4.14) it can be seen that $\Phi_{1}$ can be written as

$$
\Phi_{1}=\frac{\mu^{3} \eta}{E^{(0)}+P^{(0)}} \frac{\kappa}{48 \pi G_{5}} .
$$

We can now compare it with the expression derived earlier for this paper for $\Phi_{1}$ for charge fluid with an anomaly which is given by

$$
\Phi_{1}=\frac{2}{3} \frac{\mu^{3} \eta}{E^{(0)}+P^{(0)}} C .
$$


Therefore we obtain

$$
C=\frac{\kappa}{32 \pi G_{5}} .
$$

This is precisely the relation between the Chern-Simons coefficient and $C$ obtained directly using equations of motion in (4.19). This serves as a check for the relation between the transport coefficient $\Phi_{1}$ and the anomaly coefficient derived in this paper.

We will now use the holographic value of $\Phi_{1}$ given in (4.20) to evaluate its contribution to the chiral dispersion coefficient for the Riesner-Nordström black hole

$$
D=\frac{\sqrt{3} Q^{3} \kappa}{4 M^{2} R^{2}}+\frac{1}{2\left(E^{(0)}+P^{(0)}\right)} \Phi_{12}
$$

By the AdS/CFT correspondence the chiral dispersion relation corresponds to the quasinormal modes seen in the shear channel of the graviton fluctuations [19]. We therefore compare this dispersion coefficient with that obtained in [12] by studying the quasi-normal modes in the shear sector. They find that the dispersion coefficient is given by

$$
D^{Q N M}=\frac{\kappa^{S Y}\left(Q^{S Y}\right)^{3}}{8 m^{2} R^{3}}
$$

By comparing the action and the background solution given in [12] to that given in (4.8) and (4.10) we obtain the following relations between the variables of [12] and that used here

$$
\kappa^{S Y}=\frac{2 \kappa}{3}, \quad\left(Q^{S Y}\right)^{2}=3 q^{2}=3 Q^{2} R^{6} .
$$

Substituting the above relations in (4.25) we see that

$$
D^{Q N M}=\frac{\sqrt{3} Q^{3} \kappa}{4 M^{2} R^{2}}
$$

Since $D^{Q N M}$ must be equal to $D$ evaluated in (4.24) we see that we must have $\Phi_{12}=0$ for this system.

\section{Conclusions}

We have used the equilibrium partition function to obtain expressions for 7 parity odd transport coefficients which occur at 2 nd order for a non-conformal fluid with a single conserved charge. These transport coefficients can be expressed in terms of the anomaly, shear viscosity, bulk viscosity, charge diffusivity and thermodynamic functions. Out of these 2 transport coefficients can also be derived using the Kubo formulae. These formulae agree with that obtained by the partition function method. The equilibrium partition function also gives 3 constraints for 5 other parity odd transport coefficients at this order. The transport coefficient $\Phi_{1}$ affects chiral dispersion relations [13].

As we have mentioned earlier, parity odd coefficients have be examined earlier for conformal charge transport in [13]. There the principle used was that these coefficients should not contribute to entropy production. In general the constraints obtained by examining the zero entropy production condition will be more than that obtained from the equilibrium 
partition function. It will be interesting to carry out the analysis of [13] to non-conformal fluids and compare with the results obtained in this paper.

Our analysis of these transport properties were motivated by the possibility of studying them in holography. While this work was being done we received preprint [18] which evaluates all 2nd order transport coefficients for a charged conformal fluid in the framework of AdS/CFT. It is useful to compare the relations we have obtained for the parity odd sector with the expressions of [18].

Finally it will be useful to develop Kubo like expressions for all the parity odd transport coefficients. From the constitutive relations it seems that the remaining transport coefficients involves 3 point functions. Determining these relations will provide an alternate method to obtain the transport coefficients from holography.

\section{A Classification of parity odd data at 2 nd order in derivatives}

In this appendix we provide some details which led to the classification of the parity odd data at second order in derivatives given in table 2. We consider the following basis of vectors to construct the the second order terms:

- Parity odd vectors:

$$
\text { Vorticity : } l^{\mu}=\epsilon^{\mu \nu \alpha \beta} u_{\nu} \partial_{\alpha} u_{\beta} \quad \text { Magnetic field : } B^{\mu}=\frac{1}{2} \epsilon^{\mu \nu \alpha \beta} u_{\nu} \mathcal{F}_{\alpha \beta} .
$$

- Parity even vectors

$$
\begin{array}{r}
u^{\mu}, \quad \partial_{\mu} T, \quad \partial_{\mu} \nu, \\
\text { Electric field }: \rightarrow V^{\mu}=\frac{E^{\mu}}{T}-P^{\mu \rho} \partial_{\rho} \nu .
\end{array}
$$

Among these vectors, the electric field $V^{\mu}$ vanishes on the equilibrium fluid configuration given in (2.1). We also consider the shear tensor

$$
\sigma_{\mu \nu}=\nabla_{\langle\mu} u_{\nu\rangle} .
$$

and the scalar

$$
\Theta=\nabla_{\mu} u^{\mu}
$$

Note that both $\sigma_{\mu \nu}$ and the scalar $\Theta$ vanishes on the equilibrium configuration in (2.1). We use these basic quantities we can construct the various parity odd terms that occur at second order in derivatives given in table 2. These terms are independent and cannot be related to each other by equations of motion to first order in derivatives.

Let us consider the scalars listed in table 2: one would have thought that scalars of the form $\nabla_{\mu} l^{\mu}$ and $\nabla_{\mu} B^{\mu}$ should be listed. But it can be shown that these scalars can be related to the ones listed in the table by calculations which lead to the following identities.

$$
\begin{aligned}
\nabla_{\mu} l^{\mu} & =\left(\frac{2 q T}{E+P}\right) V^{\mu} l_{\mu}-2\left(\frac{\partial_{\mu} T}{T}\right) l^{\mu} \\
\nabla_{\mu} B^{\mu} & =\left(\frac{q T}{E+P}\right) V^{\mu} B_{\mu}-\left(\frac{\partial_{\mu} T}{T}\right) B^{\mu}-T l^{\mu}\left(V_{\mu}-\partial_{\mu} \nu\right)
\end{aligned}
$$

These identities can be verified by straight forward calculations. 
Let us now consider the vectors listed in table 2: note that the vectors $\mathcal{V}_{(3)}^{\mu}$ to $\mathcal{V}_{(9)}^{\mu}$ vanish on the equilibrium configuration (2.1). Again one would have naively expected to list terms such as $P_{\alpha}^{\mu}(u . \nabla) B^{\alpha}$. We will show now using the equations of motion to the zeroth order that this term is related to the vectors already listed in table 2. The manipulations are as follows:

$$
\begin{aligned}
P_{\alpha}^{\mu}(u . \nabla) B^{\alpha}= & \frac{1}{2} P_{\alpha}^{\mu} u^{\theta} \nabla_{\theta}\left[\epsilon^{\alpha \nu \lambda \beta} u_{\nu} \mathcal{F}_{\lambda \beta}\right], \\
= & \frac{1}{2} P_{\alpha}^{\mu} \epsilon^{\alpha \nu \lambda \beta} \mathfrak{a}_{\nu} \mathcal{F}_{\lambda \beta}+\frac{1}{2} \epsilon^{\mu \nu \lambda \beta} u_{\nu} u^{\theta} \nabla_{\theta} \mathcal{F}_{\lambda \beta}, \\
= & -\epsilon^{\mu \nu \lambda \beta} \mathfrak{a}_{\nu} u_{\lambda} u^{\theta} \mathcal{F}_{\theta \beta}-\epsilon^{\mu \nu \lambda \beta} u_{\nu} u^{\theta} \nabla_{\lambda} \mathcal{F}_{\beta \theta}, \\
= & \epsilon^{\mu \nu \lambda \beta} \mathfrak{a}_{\nu} u_{\lambda} E_{\beta}-\epsilon^{\mu \nu \lambda \beta} u_{\nu} \nabla_{\lambda} E_{\beta}+\epsilon^{\mu \nu \lambda \beta} u_{\nu}\left(\nabla_{\lambda} u^{\theta}\right) \mathcal{F}_{\beta \theta}, \\
= & -T \epsilon^{\mu \nu \alpha \beta} u_{\nu} \nabla_{\alpha} V_{\beta}+\sigma^{\mu \nu} B_{\nu}-\Theta\left[\frac{2 B^{\mu}}{3}-T\left(s \frac{\partial \nu}{\partial s}+q \frac{\partial \nu}{\partial q}\right) l^{\mu}\right] \\
& -\left(\frac{q T^{2}}{E+P}\right) \epsilon^{\mu \nu \lambda \sigma} u_{\nu} V_{\lambda} \partial_{\sigma} \nu-\frac{1}{2} \epsilon^{\mu \nu \lambda \sigma} u_{\nu} l_{\lambda} B_{\sigma}, \\
= & -T \epsilon^{\mu \nu \alpha \beta} u_{\nu} \nabla_{\alpha} V_{\beta}+\mathcal{V}_{7}^{\mu}-\frac{2 \mathcal{V}_{6}^{\mu}}{3}+T\left(s \frac{\partial \nu}{\partial s}+q \frac{\partial \nu}{\partial q}\right) \mathcal{V}_{3}^{\mu} \\
& +\left(\frac{q T^{2}}{E+P}\right) \mathcal{V}_{(9)}^{\mu}+\frac{1}{2} \mathcal{V}_{(1)}^{\mu} .
\end{aligned}
$$

Here $\mathfrak{a}_{\nu}=(u . \nabla) u_{\nu}$. In the r.h.s. of equation (A.7), all terms except the last one vanishes at equilibrium. Therefore $(u . \nabla) B^{\mu}$ does not vanish in equilibrium but it can be related to all the other vectors listed in the table. A similar analysis can be done for the vector of the form $P_{\alpha}^{\mu}(u . \nabla) l^{\alpha}$. We have

$$
\begin{aligned}
P_{\alpha}^{\mu}(u . \nabla) l^{\alpha}= & R \epsilon^{\mu \nu \alpha \beta} u_{\nu} \nabla_{\alpha} V_{\beta}+\left(\frac{\partial R}{\partial T}\right) \epsilon^{\mu \nu \alpha \beta} u_{\nu}\left(\nabla_{\alpha} T\right) V_{\beta} \\
& +\left(\frac{\partial R}{\partial \nu}\right) \epsilon^{\mu \nu \alpha \beta} u_{\nu}\left(\nabla_{\alpha} \nu\right) V_{\beta}+\sigma^{\mu \nu} l_{\nu}+\left(\frac{s}{T} \frac{\partial \nu}{\partial s}+\frac{q}{T} \frac{\partial \nu}{\partial q}-\frac{2}{3}\right) \Theta l^{\mu},
\end{aligned}
$$

where

$$
R=\frac{q T}{E+P}
$$

From equation (A.8) it is clear that $P_{\alpha}^{\mu}(u . \nabla) l^{\alpha}$ vanishes at equilibrium and that it is related to the other vectors listed in table 2 .

In fact using symmetries we can list out all the independent terms appearing at the second order. We can show that out at two derivatives either involving only the fluid variables or the velocity and derivative of the field strength, only one pseudo vector can be constructed if we demand on-shell independence for all the terms. This makes it clear that once we have chosen $\epsilon^{\mu \nu \alpha \beta} u_{\nu} \nabla_{\alpha} V_{\beta}$, all other two derivative pseudo vectors of the form mentioned earlier must be related to $\mathcal{V}_{(4)}^{\mu}$ by equations of motion. From this argument it is possible to observe that it is not necessary to list $(u . \nabla) B^{\mu},(u . \nabla) l^{\mu}, \nabla_{\mu} l^{\mu}$ or $\nabla_{\mu} B^{\mu}$ as independent data. A similar analysis can be performed for the Pseudo-tensor. This leads to the the 12 tensors listed in 2 . 


\section{B Consistency of the fluid profiles at the linear order}

In this section we show that the velocity perturbations and the background field configuration considered in equations (3.1) to (3.4) consistently solve the linearized fluid equations of motion without the need of the any other fluctuations. The perturbations depends only on the time, $t$ and the spatial direction, $y$.

We will consider the perturbations to the linear order. We first write down all the components of the stress tensor to 2 nd order in derivatives and to linear order in the perturbations. This are given by

$$
\begin{aligned}
T^{t t}= & E^{(0)} \\
T^{t x}= & =\left(E^{(0)}+P^{(0)}\right) v^{x}+P^{(0)} h_{t x}, \\
T^{t z}= & \left(E^{(0)}+P^{(0)}\right) v^{z}+P^{(0)} h_{t z}, \\
T^{t y}= & 0, \\
T^{x x}= & P^{(0)}, \\
T^{x y}= & -P^{(0)} h_{y x}-\eta\left(\partial_{y} v^{x}+\partial_{t} h_{y x}\right) \\
& +\frac{1}{2} \Phi_{1} \partial_{y}^{2}\left(v^{z}+h_{z t}\right)+\frac{1}{2} \Phi_{2} \partial_{y}^{2} a_{z} \\
& +\frac{1}{2} \Phi_{12} \partial_{y}\left(\partial_{y} v^{z}+\partial_{t} h_{y z}\right), \\
T^{x z}= & 0, \\
T^{y y}= & P^{(0)}, \\
T^{y z}= & -P^{(0)} h_{y z}-\eta\left(\partial_{y} v^{z}+\partial_{t} h_{y z}\right) \\
& -\frac{1}{2} \Phi_{1} \partial_{y}^{2}\left(v^{x}+h_{z t}\right)-\frac{1}{2} \Phi_{2} \partial_{y}^{2} a_{x} \\
& -\frac{1}{2} \Phi_{12} \partial_{y}\left(\partial_{y} v^{x}+\partial_{t} h_{y}\right), \\
T^{z z}= & P^{(0)} .
\end{aligned}
$$

Now there are 4 equations of motion for the stress tensor. We will show that that the $t$ and $y$ components are trivially satisfied. The $x$ and $z$ components of this equations can be used to determine the velocity profiles. The $t$ component of the equations of motion of the stress tensor is given by

$$
\partial_{t} T^{t t}+\partial_{y} T^{y t}+\Gamma_{\mu t}^{\mu} T^{t t}+\Gamma_{t t}^{t} T^{t t}+\Gamma_{x x}^{t} T^{x x}+\Gamma_{y y}^{t} T^{y y}+\Gamma_{z z}^{t} T^{z z}=0 .
$$

Note that we have used the fact that all Christofell symbols are first order in the fields. We have also used the fact that the only dependence is through time $t$ and $y$. Now examining the Christofell symbols given in (3.5) we see that there is no contribution to the equation in (B.2) from any term involving the Christofell symbols. Also since $T^{t t}=E^{(0)}$, the first term also vanishes. The second term in the equation vanishes because $T^{y t}=0$. Thus this equation is satisfied and imposes no constrains on the velocity configuration chosen. Now let examine the $y$ component of the equations of motion of the stress tensor. We have

$$
\partial_{t} T^{t y}+\partial_{y} T^{y y}+\Gamma_{\mu y}^{\mu} T^{y y}+\Gamma_{t t}^{y} T^{t t}+\Gamma_{x x}^{y} T^{x x}+\Gamma_{y y}^{y} T^{y y}+\Gamma_{z z}^{y} T^{z z}=0 .
$$


Again, examining each term one can see that this equation is also satisfied. Thus the $t$ and $y$ equations are satisfied. Thus $v_{x}$ and $v_{y}$ are determined by the $x$ and $z$ component of the equations of motion

$$
\begin{aligned}
\partial_{t} T^{t x}+\partial_{t} h_{t x} T^{t t}+\partial_{y} T^{y x} & =0, \\
\partial_{t} T^{t z}+\partial_{t} h_{t z} T^{t t}+\partial_{y} T^{y z} & =0 .
\end{aligned}
$$

To complete the analysis we show that the equations of motion of the charge current are also trivially satisfied. The only non-zero values of the the vorticity to the linear order is given by

$$
l^{x}=\partial_{y} v_{z}, \quad l^{z}=-\partial_{y} v_{x} .
$$

Similarly the non-zero values of the magnetic field to the linear order

$$
B^{x}=\frac{1}{2} \partial_{y} a_{z}, \quad B^{z}=-\frac{1}{2} \partial_{y} a_{x} .
$$

One can also see that for the background in (3.1) and fluctuations to the linear order the electric field $V^{\mu}$ vanishes to the linear order in the fields. It can also be seen that to the linear order in fields the vectors $\mathcal{V}_{(i)}^{\mu}$ with $i=1, \cdots 9$ all vanish. Now using all this information, the currents to the linear order in fields and to second order in derivatives are given by

$$
\begin{aligned}
& J^{t}=-q^{(0)}, \\
& J^{x}=q^{(0)} v^{x}+\xi l^{x}+\xi_{B} B^{x}, \\
& J^{y}=0, \\
& J^{z}=q^{(0)} v^{z}+\xi l^{z}+\xi_{B} B^{z} .
\end{aligned}
$$

Now the current conservation equation to this order reads

$$
\partial_{t}\left(\sqrt{g} J^{t}\right)+\partial_{y}\left(\sqrt{g} J^{y}\right)=0,
$$

and since $\sqrt{g}$ does not change to the linear order, this equation is satisfied and does not impose any further conditions on the velocities $v^{x}$ and $v^{y}$. Here we have used that the only dependence is through $t$ and $y$.

Thus the constant background with the linear velocity profiles $v^{x}, v^{y}$ given in (3.1) to (3.4) consistently solve the equations of motion. There are only 2 equations which determine the velocity profiles $v^{x}, v^{y}$. A simple way of stating this is that we have turned only the shear fluctuations which decouple from the rest of the modes.

\section{Acknowledgments}

We thank Aninda Sinha for organizing a stimulating workshop on "Non-perturbative gauge theories, holography and all that" which initiated this collaboration. We thank Nabamita Banarjee, Bobby Ezuthachan, Yashodhan Hatwalne, Sachin Jain, Shiraz Minwalla and Rahul Pandit for useful and stimulating discussions. S.B thanks the CHEP, IISc and the ICTS-TIFR for hospitality during the early phase of this work. The work of J.R.D is partially supported by the Ramanujan fellowship DST-SR/S2/RJN-59/2009. 
Open Access. This article is distributed under the terms of the Creative Commons Attribution License (CC-BY 4.0), which permits any use, distribution and reproduction in any medium, provided the original author(s) and source are credited.

\section{References}

[1] D.T. Son and P. Surowka, Hydrodynamics with Triangle Anomalies, Phys. Rev. Lett. 103 (2009) 191601 [arXiv:0906.5044] [INSPIRE].

[2] N. Banerjee, J. Bhattacharya, S. Bhattacharyya, S. Dutta, R. Loganayagam et al., Hydrodynamics from charged black branes, JHEP 01 (2011) 094 [arXiv:0809.2596] [INSPIRE].

[3] J. Erdmenger, M. Haack, M. Kaminski and A. Yarom, Fluid dynamics of R-charged black holes, JHEP 01 (2009) 055 [arXiv:0809.2488] [INSPIRE].

[4] R. Loganayagam and P. Surowka, Anomaly/Transport in an Ideal Weyl gas, JHEP 04 (2012) 097 [arXiv: 1201.2812] [INSPIRE].

[5] K. Jensen, Triangle Anomalies, Thermodynamics and Hydrodynamics, Phys. Rev. D 85 (2012) 125017 [arXiv: 1203.3599] [INSPIRE].

[6] N. Banerjee, S. Dutta, S. Jain, R. Loganayagam and T. Sharma, Constraints on Anomalous Fluid in Arbitrary Dimensions, JHEP 03 (2013) 048 [arXiv: 1206.6499] [INSPIRE].

[7] R. Loganayagam, Anomalies and the Helicity of the Thermal State, JHEP 11 (2013) 205 [arXiv: 1211.3850] [INSPIRE].

[8] V. Nair, R. Ray and S. Roy, Fluids, Anomalies and the Chiral Magnetic Effect: A Group-Theoretic Formulation, Phys. Rev. D 86 (2012) 025012 [arXiv:1112.4022] [INSPIRE].

[9] K. Landsteiner, E. Megias and F. Pena-Benitez, Anomalous Transport from Kubo Formulae, Lect. Notes Phys. 871 (2013) 433 [arXiv:1207.5808] [INSPIRE].

[10] P. Romatschke and D.T. Son, Spectral sum rules for the quark-gluon plasma, Phys. Rev. D 80 (2009) 065021 [arXiv:0903.3946] [INSPIRE].

[11] A.V. Andreev, D.T. Son and B. Spivak, Hydrodynamics of liquids of chiral molecules and suspensions containing chiral particles, Phys. Rev. Lett. 104 (2010) 198301.

[12] B. Sahoo and H.-U. Yee, Holographic chiral shear waves from anomaly, Phys. Lett. B 689 (2010) 206 [arXiv:0910.5915] [INSPIRE].

[13] D.E. Kharzeev and H.-U. Yee, Anomalies and time reversal invariance in relativistic hydrodynamics: the second order and higher dimensional formulations, Phys. Rev. D 84 (2011) 045025 [arXiv: 1105.6360] [INSPIRE].

[14] N. Banerjee et al., Constraints on Fluid Dynamics from Equilibrium Partition Functions, JHEP 09 (2012) 046 [arXiv: 1203.3544] [INSPIRE].

[15] K. Jensen et al., Towards hydrodynamics without an entropy current, Phys. Rev. Lett. 109 (2012) 101601 [arXiv:1203.3556] [INSPIRE].

[16] K. Jensen, R. Loganayagam and A. Yarom, Thermodynamics, gravitational anomalies and cones, JHEP 02 (2013) 088 [arXiv:1207.5824] [INSPIRE].

[17] I. Amado, K. Landsteiner and F. Pena-Benitez, Anomalous transport coefficients from Kubo formulas in Holography, JHEP 05 (2011) 081 [arXiv:1102.4577] [INSPIRE]. 
[18] E. Megias and F. Pena-Benitez, Holographic Gravitational Anomaly in First and Second Order Hydrodynamics, JHEP 05 (2013) 115 [arXiv: 1304.5529] [INSPIRE].

[19] P.K. Kovtun and A.O. Starinets, Quasinormal modes and holography, Phys. Rev. D 72 (2005) 086009 [hep-th/0506184] [INSPIRE]. 